\title{
anthropozoologica
}

$2019 \cdot 54 \cdot 6$

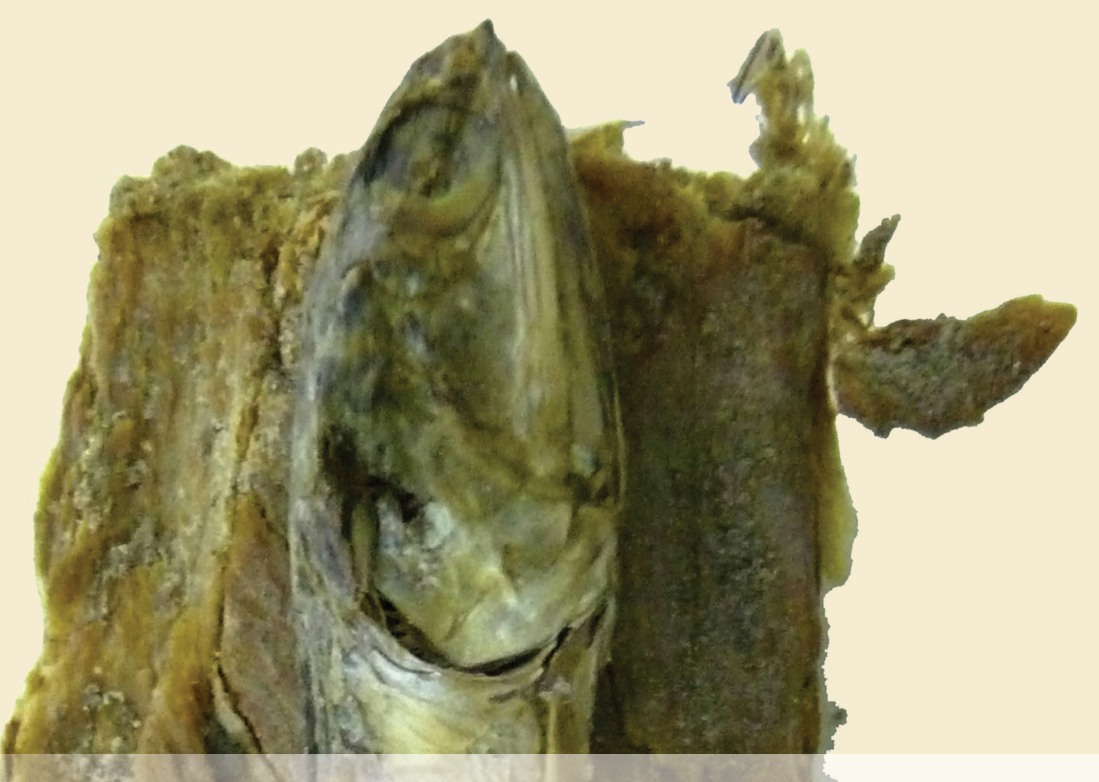

The ways of fish beyond the sea: fish circulation and consumption in the Atacama desert, northern Chile, during the Formative period (500 cal B.C.-700 cal A.D.) 
DiRECTEUR DE LA PUBLICATION: Bruno David,

Président du Muséum national d'Histoire naturelle

RÉDACTRICE EN CHEF / EDITOR-IN-CHIEF: Joséphine Lesur

RÉDACTRICE / EDITOR: Christine Lefèvre

Responsable des Actualités SCIENTIFIQUes / Responsible fOR SCIENTIFIC NEWS: Rémi Berthon

ASSISTANTE DE RÉDACTION / ASSISTANT EDITOR: Emmanuelle Rocklin (anthropo@mnhn.fr)

MISE EN PAGE / PAGE LAYOUT: Emmanuelle Rocklin, Inist-CNRS

COMITÉ SCIENTIFIQUE / SCIENTIFIC BOARD:

Cornelia Becker (Freie Universität Berlin, Berlin, Allemagne)

Liliane Bodson (Université de Liège, Liège, Belgique)

Louis Chaix (Muséum d'Histoire naturelle, Genève, Suisse)

Jean-Pierre Digard (CNRS, Ivry-sur-Seine, France)

Allowen Evin (Muséum national d'Histoire naturelle, Paris, France)

Bernard Faye (Cirad, Montpellier, France)

Carole Ferret (Laboratoire d'Anthropologie Sociale, Paris, France)

Giacomo Giacobini (Università di Torino, Turin, Italie)

Véronique Laroulandie (CNRS, Université de Bordeaux 1, France)

Marco Masseti (University of Florence, Italy)

Georges Métailié (Muséum national d'Histoire naturelle, Paris, France)

Diego Moreno (Università di Genova, Gènes, Italie)

François Moutou (Boulogne-Billancourt, France)

Marcel Otte (Université de Liège, Liège, Belgique)

Joris Peters (Universität München, Munich, Allemagne)

François Poplin (Muséum national d'Histoire naturelle, Paris, France)

Jean Trinquier (École Normale Supérieure, Paris, France)

Baudouin Van Den Abeele (Université Catholique de Louvain, Louvain, Belgique)

Christophe Vendries (Université de Rennes 2, Rennes, France)

Noëlie Vialles (CNRS, Collège de France, Paris, France)

Denis Vialou (Muséum national d'Histoire naturelle, Paris, France)

Jean-Denis Vigne (Muséum national d'Histoire naturelle, Paris, France)

Arnaud Zucker (Université de Nice, Nice, France)

COUVERTURE / COVER:

Aliment trouvé dans le cimetière Topater 01, composé de deux types de poissons attachés par une corde tressée: un anchois complet et un filet désossé d'un plus gros poisson. Photographie: F. Gallardo. / Composite food found in Topater 01 cemetery, made of two kind of fishes tied together by a braided rope: a complete anchoveta and a boneless fillet of a bigger fish. Photograph: F. Gallardo.

Anthropozoologica est indexé dans / Anthropozoologica is indexed in:

- Social Sciences Citation Index

- Arts \& Humanities Citation Index

- Current Contents - Social \& Behavioral Sciences

- Current Contents - Arts \& Humanities

- Zoological Record

- BIOSIS Previews

- Initial list de l'European Science Foundation (ESF)

- Norwegian Social Science Data Services (NSD)

- Research Bible

Anthropozoologica est distribué en version électronique par / Anthropozoologica is distributed electronically by:

- BioOne ${ }^{\circledR}$ (http://www.bioone.org)

Anthropozoologica est une revue en flux continu publiée par les Publications scientifiques du Muséum, Paris, avec le soutien du CNRS.

Anthropozoologica is a fast track journal published by the Museum Science Press, Paris, with the support of the CNRS.

Les Publications scientifiques du Muséum publient aussi / The Museum Science Press also publish:

Adansonia, Zoosystema, Geodiversitas, European Journal of Taxonomy, Naturae, Cryptogamie sous-sections Algologie, Bryologie, Mycologie.

Diffusion - Publications scientifiques Muséum national d'Histoire naturelle

CP 41 - 57 rue Cuvier F-75231 Paris cedex 05 (France)

Tél.: 33 (0)1 40794805 / Fax: 33 (0)1 40793840

diff.pub@mnhn.fr / http://sciencepress.mnhn.fr

(C) Publications scientifiques du Muséum national d'Histoire naturelle, Paris, 2019

ISSN (imprimé / print): 0761-3032 / ISSN (électronique / electronic): 2107-08817 


\section{The ways of fish beyond the sea: fish circulation and consumption in the Atacama desert, northern Chile, during the Formative period (500 cal B.C.-700 cal A.D.)}

Benjamín BALLESTER

UMR7041 Archéologies et Sciences de l'Antiquité (ArScAN), Équipe Ethnologie préhistorique, CNRS, Université Paris 1 Panthéon-Sorbonne, Université Paris Nanterre, Ministère de la Culture,

21, Allée de l'Université, F-92023 Nanterre Cedex (France) benjaminballesterr@gmail.com

Elisa CALÁs

Programa de Doctorado en Arqueología Universidad de Buenos Aires, Puan 430, CABA, C1406CQJ (Argentina) elisa.calas@gmail.com

Rafael LABARCA Escuela de Arqueología, Universidad Austral de Chile, Sede Puerto Montt, Los Pinos s/n, Puerto Montt (Chile) r.labarca.e@gmail.com

William PESTLE

Department of Anthropology, University of Miami, 5202 George E Merrick St, Coral Gables, FL-33146 (United States)

w.pestle@miami.edu

Francisco GALLARDO

Centro de Estudios Interculturales e Indígenas (CIIR), Pontificia Universidad Católica de Chile, Av. Vicuña Mackenna 4860, Campus San Joaquín, Antropología UC,

2 do piso Macul, Santiago 7820436 (Chile) fgallardo.ibanez@gmail.com

Claudia CASTILLO

Independent researcher claucasbat@gmail.com

Gonzalo PIMENTEL

Fundación Patrimonio Desierto de Atacama, Santa Victoria 45, Santiago de Chile (Chile) gpimentel@desiertoatacama.com

Cristobal OYARZO

Independent researcher cristobaloyarzo@gmail.com

Ballester B., Calás E., Labarca R., Pestle W., Gallardo F., Castillo C., Pimentel G. \& Oyarzo C. 2019. - The ways of fish beyond the sea: fish circulation and consumption in the Atacama desert, northern Chile, during the Formative period (500 cal B.C.-700 cal A.D.). Anthropozoologica 54 (6): 55-76. https://doi.org/10.5252/anthropozoologica2019v54a6. http://anthropozoologica.com/54/6 
KEY WORDS

Dried fish,

exchange,

culinary,

stable isotopes,

ichthyology,

Formative period.

\section{MOTS CLÉS}

Poisson séché,

échange,

culinaire,

isotopes stables,

ichtyologie, période Formative.

\begin{abstract}
Along the Atacama Desert coast, fish has always been a staple food and by the Formative period $(500 \mathrm{cal} \mathrm{B.C.}-700 \mathrm{cal}$ A.D.) it had become a product in high demand by the inhabitants of the inland valleys, oases and ravines of the desert. In this paper we explore the technologies used in coastal fishing activities, the diverse species caught, and fish processing and preserving techniques. We further examine the circulation routes of the product through the desert and associated strategies, the agents involved in transporting it and consumption levels in inland villages. Our study employs a multivariate analysis that includes evidence from zooarchaeology, stable isotope analysis of deceased individuals, and the composition of human coprolites, all of which were recovered from domestic waste, funerary contexts, and rest stops associated with the circulation routes running between the coast and the inland desert regions. Our results suggest that in this ancient social context, food was not only used to quell hunger, but through its associated economic cycles of production, circulation and consumption, was part of a complex and extended web of social relations. Within that network, food functioned as material culture, and as such enabled social distinctions to emerge within local groups and cultural negotiations to be conducted among different localities. Fish circulation and consumption played an active role in the reproduction of a social structure characterized by close and firm ties between marine hunter-fisher-gatherers and agropastoral communities, despite their long distance from each other.
\end{abstract}

\begin{abstract}
RÉSUMÉ
La route du poisson au-delà de la mer: circulation et consommation de poisson dans le désert d'Atacama, au nord du Chili, durant la période Formative (500 av. J.-C.-700 apr. J.-C.).

Le long de la côte du désert d'Atacama, le poisson a toujours été un aliment de base et dès la période Formative (500 av. J.-C.-700 apr. J.-C.), il devint un produit très convoité par les habitants des vallées, oasis et ravins des terres intérieures du désert. Dans cet article, nous explorons les technologies employées dans la pêche côtière, la diversité des espèces capturées et les techniques de traitement et de conservation du poisson. Nous examinons ensuite les voies de circulation de ce produit à travers le désert, ainsi que les stratégies et les agents associés dans son transport. Enfin, nous évaluons sa consommation dans les villages intérieurs. Notre étude utilise une analyse multivariée incluant des éléments issus de la zooarchéologie, l'analyse isotopique stable d'individus décédés et la composition des coprolithes humains, provenant tous de sites domestiques et funéraires, ainsi que de sites de repos temporaires associés aux voies de circulation entre la côte et l'intérieur du désert. Nos résultats suggèrent que, dans ce vaste contexte social et historique, les aliments ont non seulement été employés pour apaiser la faim, mais qu'à travers les différents cycles économiques de production, circulation et consommation qui leur sont liés, ils ont également intégré un réseau complexe de rapports sociaux. Dans ce cadre, les aliments auraient fonctionné comme une culture matérielle et, en tant que telle, auraient contribué à l'émergence de distinctions sociales et à l'établissement de négociations culturelles entre les différentes populations locales. La circulation et la consommation de poisson jouaient ainsi un rôle actif dans la reproduction d'une structure sociale caractérisée par des liens étroits et fermes entre les collectifs de chasseurs-pêcheurs-cueilleurs du littoral et les communautés agropastorales du désert intérieur, malgré leur éloignement géographique.
\end{abstract}

\section{INTRODUCTION}

According to the Glosario de la lengua atacameña compiled by Emilio Vaïsse et al. (1896: 16), among the people of the Atacama Desert valleys and oases the word "ckackchi" was used interchangeably to refer both to "fish" and as a descriptor for any food that was "good, pleasant, and tasty to the palate". More than a linguistic coincidence, the double meaning of the word "ckackchi" shows clearly the level of respect and affinity that groups of the Atacama interior had for this foreign product.

However, the history of such dietary predilections can be difficult to reconstruct with any scientific certainty by archaeological means alone, as it requires discerning subtle aspects of personal and group appreciation that do not leave material traces. What archaeologists can hope to elucidate instead are aspects of the production, circulation, and consumption of these highly valued foods. In the present case, evidence attesting to these processes includes the preserved traces of exchange routes, the waste middens of habitation sites, human coprolites, funerary food offerings, and the stable isotope composition of human osseous remains.

The earliest evidence of fish consumption in the archaeological record of the Atacama interior indicates that this product was being consumed, in small quantities at first, between 4000-2000 cal B.C. in some inland valley and oasis sites, including Chiuchiu, San Pedro de Atacama, and Tarapacá 


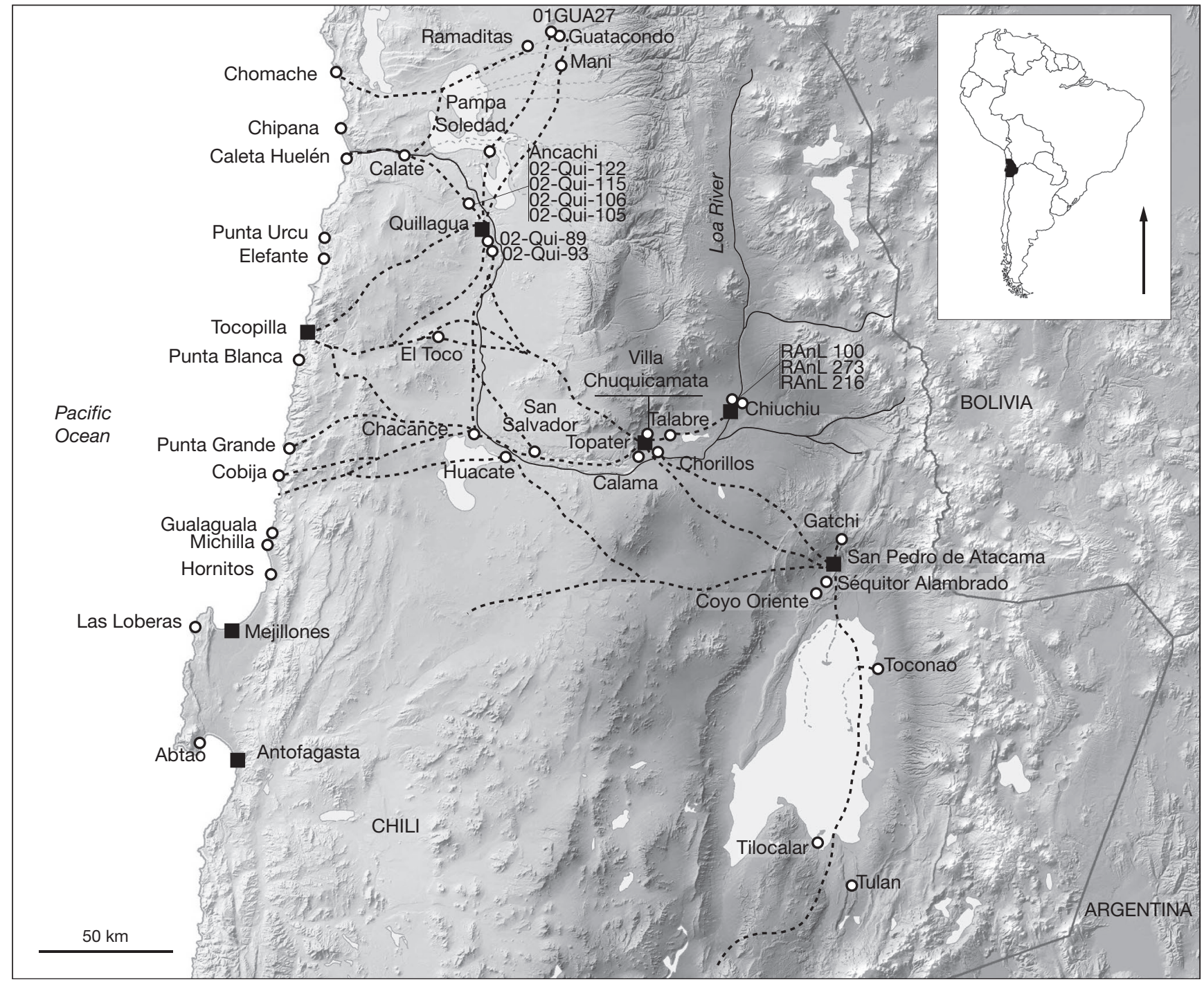

FIG. 1. - Atacama Desert (Chile) map with the localities mentioned.

(Druss 1978; Casteel 1980; Benavente 1988-1989; Cartajena 1994; Agüero \& Uribe 2011; Fig. 1). During the coastal Formative period $(500 \mathrm{cal}$ B.C.-700 cal A.D.), however, the inland circulation and consumption of fish intensified, evincing a burgeoning system of regional exchange (Casteel 1980; Follet 1980; Scott et al. 2005; Castillo 2011, 2015; Santana et al. 2012; Torres-Rouff et al. 2012a; Pestle et al. 2015a, b; Castillo et al. 2017; Pestle 2017).

In this article, we offer a preliminary model of fish consumption in the Atacama desert interior during the Formative period (500 cal B.C.-700 cal A.D.) employing zooarcheological, bioanthropological, and paleofecal information (both published and unpublished). In particular, we focus on such aspects as the richness and relative frequency of the fish species consumed, the techniques required to preserve them for exchange, and the relationship between distance from the coast and fish consumption. These three aspects shed light upon the human agents involved in the circulation and ex- change, the technologies and strategies they employed, and the different social contexts in which this food was consumed in the interior valleys and oases of the Atacama desert. In this perspective, we consider fish to be not simply a food but a good, an element of material culture (Van der Veen 2003) whose value goes beyond its merely nutritive value, as it plays an active role in more complex and diverse aspects of the social, cultural, political and economic spheres (Hastorf 2012).

\section{ATACAMA DURING THE FORMATIVE PERIOD}

The Atacama Desert is one of the most arid regions on the planet, with very low precipitation and much lower than average primary productivity in general; however, due to a pronounced east-west altitudinal gradient (Fig. 2), the region actually comprises a diverse range of ecological niches with characteristic biota and different degrees of primary 
productivity (Weischet 1975; Ortlieb 1995; Marquet et al. 1998; McKay et al. 2003; Clarke 2006). In the pre-Hispanic period, that uneven distribution of resources forged a human geography in which the inhabitants tended to be much more concentrated in areas where resources and freshwater were more readily available (Fig. 1). Thus, in one of the driest and harshest deserts of the globe, valleys and oases watered by Andean runoff and scattered freshwater springs on the Pacific coast became the primary focus for habitation (Núnez \& Dillehay 1979; Llagostera 2013), and the intermediate spaces between those focal points remained uninhabited, even to this day in most cases (Pimentel 2012).

Those habitable locations were usually far from one another, obliging their inhabitants to travel tens and even hundreds of kilometers across difficult terrain to reach a neighboring settlement, where they could build or strengthen economic, political, and social ties, all indispensable for social reproduction (Núnez \& Dillehay 1979; Berenguer \& Pimentel 2010; Souza et al. 2010; Ballester \& Gallardo 2011; Pimentel 2012; Torres-Rouff et al. 2012a; Pimentel et al. 2017). The material remains of this process include: the ancient routes and travelrelated structures that connected archaeological localities; the remains of individuals who died in transit; the large quantity and wide range of foreign goods found at residential sites and cemeteries; and the clear similarities in aspects of culture, style, representation, and design among distant settlements, marking the flow not only of people and goods, but also of social information (Núnez 1985; Agüero et al. 2006; Cases et al. 2008; Pimentel et al. 2010, 2011; Pimentel 2012; TorresRouff et al. 2012b; Gallardo \& Cabello 2015; Carrasco et al. 2015; Labarca et al. 2015; Gallardo et al. 2017a).

During the Formative period, major social changes occurred in the Atacama Desert that were accompanied by similarly significant shifts in the economic and political structures (Castro et al. 2016). Sedentism, surplus production, and inter-community linkages became central to the societies of the region and, although those changes occurred across the region, each locality experienced them in its own particular way. In the valleys and oases of the interior, differences abounded in the scale of food gathering versus agricultural development, the consolidation of pastoralist practices, the architecture employed in building village complexes, the use of new hunting technologies, and the scale and scope of handicraft manufacturing (Gallardo 1993; Ayala 2001; Souza 2004; Agüero \& Cases 2004; Rees \& Souza 2004; Uribe \& Ayala 2004; Gallardo \& Yacobaccio 2005; Núnez et al. 2005, 2006; Agüero et al. 2006; Cartajena et al. 2007; Gallardo \& Souza 2008; Uribe 2009; Uribe \& Vidal 2012; Adán et al. 2013).

On the Pacific coast, communities maintained their way of life based on hunting, fishing, and gathering marine resources. But they also began to intensify production, developing innovative hunting and fishing techniques to increase their catches, using the surpluses to enable them to take a leading role in the exchange networks that would eventually cross the Atacama Desert (Latcham 1909; Llagostera 1989, 1990; Castelleti 2007; Ballester \& Clarot 2014; Gallardo et al. 2017a).
During this period, these marine hunter-fisher-gatherers lived in stable residential settlements and used a logistical mobility strategy in which they moved back and forth along the coast in seagoing vessels and traveled on foot into the desert interior, building an extensive territory that incorporated the waters of the Pacific, the shoreline, and the inland pampa (Cases et al. 2008; Blanco et al. 2010; Pimentel et al. 2010, 2011; Ballester \& Gallardo 2011; Ballester \& Clarot 2014; Ballester \& Crisóstomo 2017; Pimentel \& Ugarte 2017; Gallardo et al. 2017a).

Along with their residences, they built monumental cemeteries - fields that could reach up to 300 tumuli built of soil and rock of $1.5 \mathrm{~m}$ in height and $7 \mathrm{~m}$ in diameter average dimensions, most containing the remains of one individual accompanied by a set of rich grave goods - that represent a huge investment of collective labor (Latcham 1910; Capdeville 1928; Mostny 1964b; Spahni 1967; Núñez 1971; Moragas 1982; Ballester \& Clarot 2014; Gallardo et al. 2017a). Within these graves were everyday objects for obtaining marine resources such as harpoons, copper and cactus spine hooks, bone tools for extracting mollusks, and nets for fishing, among many other items. Foreign goods from the valleys and oases of the interior were also common among these grave goods and included ceramic vessels, gold and copper adornments, textiles, snuff trays, terrestrial shells used as pigment containers, foreign animals and plant-fiber baskets. All of these would have been obtained through the exchange network for surplus dried fish and shell artifacts (Latcham 1909; Mostny 1964b; Ballester \& Clarot 2014; Labarca et al. 2015; Gallardo 2017; Carrasco et al. 2017; Gallardo et al. 2017a, b).

Archeological investigation has revealed that during the Formative period, the circulation of goods between the coast and interior was in the hands of agents from both the coast and from inland valleys and oases. The former traveled inland on foot for a variety of economic and productive purposes, one of the most important being exchange (Pimentel et al. 2010, 2011; Ballester \& Gallardo 2011; Ballester \& Crisóstomo 2017; Pimentel \& Ugarte 2017; Blanco et al. 2017). At the same time, but with increasing intensity from the Formative and following periods, certain individuals would have come from the interior valleys and oases toward the coast for the same purpose - to exchange goods and stablish social interactions - but most of them would have used camelid caravans for this purpose (Núñez \& Dillehay 1979; Núnez 1985; Pimentel et al. 2010, 2011, 2017; Torres-Rouff et al. 2012b).

\section{METHODOLOGY}

The study of fish consumption in the Atacama interior relied heavily upon stable isotope analyses to determine diet (Santana 2011; Santana et al. 2012, 2015; Pestle et al. 2015a, b; Pestle 2017), although in general the information obtained has remained in the specialized realm, uncoupled from other sources of information. Our approach, in contrast, seeks to 


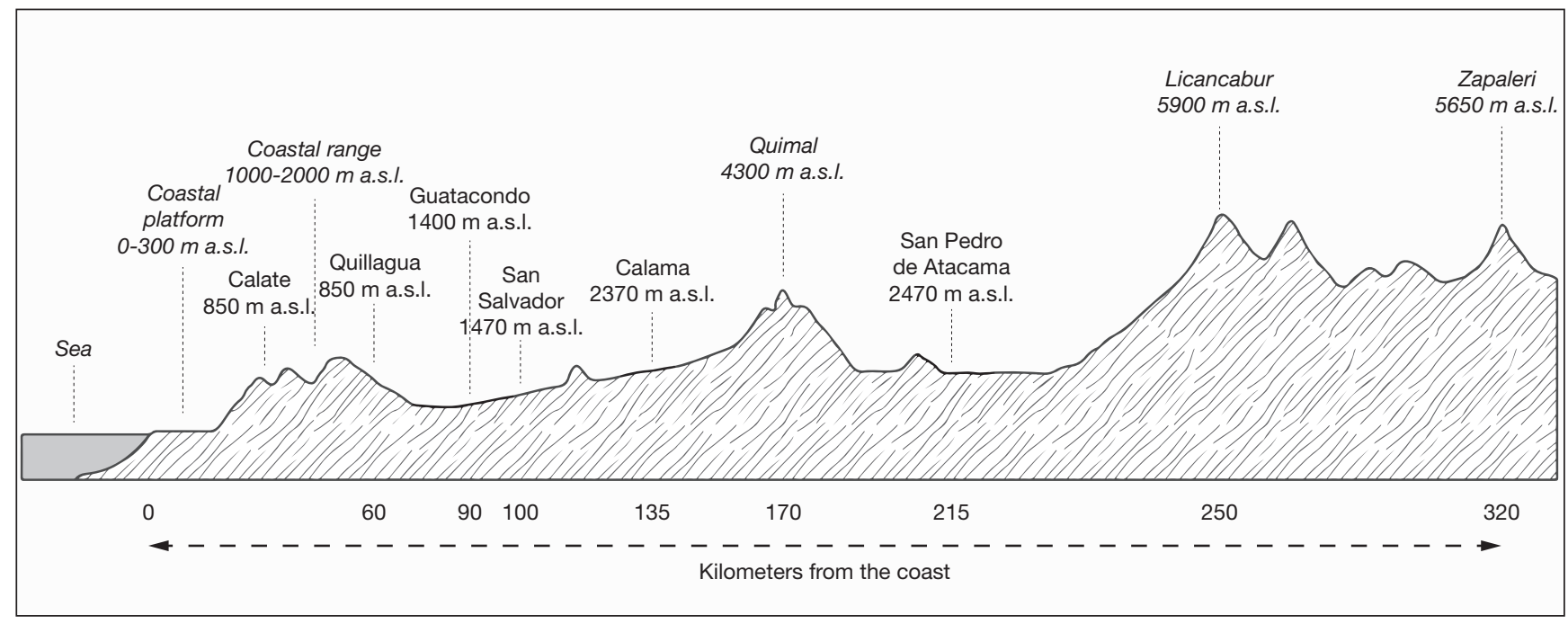

FIG. 2. - Altitudinal profile of the region with the position from the different localities mentioned in the article. Sites in italics: main geographical landmarks. Abbreviation: $\mathbf{m}$ a.s.l., meters above sea level.

link up different materialities, archeological contexts, and analytical perspectives by integrating zooarcheological and paleofecal evidence with bioanthropological data, as well as taking into account domestic, logistic and route waste, but also funerary contexts.

As a first step, we identified all archeological sites in the Atacama interior that showed evidence of fish consumption and had been dated to the Formative period $(\mathrm{N}=32)^{1}$. Eleven of these sites $(34.38 \%)$ were associated with transit routes and $21(65.63 \%)$ were in an inland valley or oasis. Our team conducted the analysis for 20 of those 32 sites $(62.5 \%)$ - most of them on transit routes $(\mathrm{N}=11)$ or in the localities of Quillagua $(\mathrm{N}=6)$, Guatacondo $(\mathrm{N}=2)$ and the middle Loa $(\mathrm{N}=1)$ - while the rest $(\mathrm{N}=12)$ were obtained from the literature. Aware of the difficulty of comparing results generated through different sampling strategies and/or interventional intensities, especially in quantitative terms, our comparisons were made on the basis of taxonomic richness (NTAXA).

Taxonomic identification for the contexts we analyzed employed both reference skeletons and osteological guides (e.g. Falabella et al. 1995), while quantification was based on the minimum number of elements (MNE) and skeletal frequency was based on the percentage of the minimum number of animal units (\%MAU) (Binford 1978; Grayson 1984). For analytical purposes, fish skeletons were divided into two sections: cranial, including facial and neurocranial bones; and postcranial, which included only the vertebral column (first vertebra included), appendicular skeleton (pectoral and pelvic girdles), and tail (Hoffman et al. 2000). Other minor bones such as spines, ribs, and pterygiophores were not considered. For stable isotopes and coprolites, data from previously published studies were used.

1. Regrettably, the faunal studies in the region are scarce, and it is very difficult to ensure the number of archaeological site that surely do not have fish remains to compare.

\section{THE CYCLE OF FISH: PRODUCTION, DISTRIBUTION, AND CONSUMPTION}

FishiNG TECHNOLOGIES DURING THE FORMATIVE PERIOD Atacama coastal groups had been practicing hook-and-line fishing for at least 4000 years before the Formative period (Mostny 1964a; Bittmann 1978, 1984; Llagostera 1989; Contreras et al. 2011; Flores et al. 2016), but that period witnessed the replacement of the shell and compound hooks used up to the end of the Late Archaic (4000-2000 cal B.C.) with hooks made of cactus needles and, beginning in 500 cal B.C., hooks made of hammered copper. These two later technologies continued to be used up until the moment of European contact (Fig. 3F-J; Mostny 1942, 1964b; Bird 1943; Spahni 1967; Boisset et al. 1969; Bravo 1981; Llagostera 1989, 1990; Castelleti 2007; Salazar et al. 2010; Ballester \& Clarot 2014; Latorre 2017). In addition to hooks, the act of fishing required at least two more artefactual components - fishing line (in this case of cotton) and line weights. The earliest cotton fishing line dates to a few centuries before $4000 \mathrm{cal} \mathrm{B.C.,}$ attesting to the use of such technology throughout the later coastal occupation sequence (Fig. 3K; Bird 1946; Boisset et al. 1969; Ballester \& Clarot 2014). For their part, line weights varied in form, size, and raw material used. The earliest examples, dating to around 5000-4000 cal B.C., were composed of rock, bone, or Choromytilus chorus (Molina, 1782) shells (Mostny 1964b; Silva \& Bahamondes 1968; Boisset et al. 1969; Núñez et al. 1974; Contreras et al. 2011; Flores et al. 2016), while those attributed to the Formative period were of stone and often cigar-shaped (Fig. 3B-E; Mostny 1964b; Spahni 1967; Ballester \& Clarot 2014).

Another fishing instrument employed during this period was the gaff or "potera", a device used to catch cephalopods and for capturing fish in large shoals (Fig. 3A; Jiménez 1943; Silva \& Bahamondes 1968; Van Kessel 1988; Ballester 2017; Labarca et al. 2017). The device can be recognized by certain 
features, particularly the grooved spherical weights and the lateral bone barbs that aided in penetrating and securing the prey (Bird 1943, 1946; Ballester \& Clarot 2014; Labarca et al. 2017). The earliest evidence for this technology comes from Late Archaic funerary contexts at Caleta Huelen 42 and Taltal (Mostny 1964b; Zlatar 1975), although it appears much more frequently in the Formative archeological contexts. Fish were also captured using harpoons, which allowed individual fish to be caught without the need for bait (Fig. 3L-N; Mostny 1964b; Llagostera 1989; Ballester 2018).

Nets are one of the least-studied fishing artifacts of the northern Chilean coast, in part owing to the very small number of surviving archaeological examples. The earliest published examples come from the site of Abato 1 (44543369 cal B.C.), where three distinct types (single link, double link, and knotted) have been identified (Boisset et al. 1969). Net-like textiles are often found in Formative period settlement sites, while complete bags and larger fragments have been recovered from some funerary contexts (Fig. 3O; Spahni 1967; Ballester \& Clarot 2014).

Overall, these finds give a picture of a fishing toolkit that satisfied many distinct needs (Ballester 2017). Each type and size of hook, when paired with a different kind of weight, targeted a certain kind of fish and could be customized according to fish habitat, size, and water depth. The gaff, thanks to its lateral barbs, could catch more than one fish at a time and was used against large shoals of fish, ensuring high efficiency but low selectivity. The harpoon could be used to catch fish cruising close to the surface, as it required the hunter to keep the prey in view to hit it with the small head, for example, thereby permitting more targeted, selective approach, but only for certain species. Nets, for their part, produced a mass of fish limited only by the size of holes in the net, its overall size, and the quantity of fish in the water, giving the fisherman an abundant catch but little selectivity.

The productiveness of these artifacts can only be measured as a function of the strategy used and how it was implemented, both of which are difficult to ascertain from the archaeological record. We do know that after European contact, long fishing lines with more than 700-800 hooks, called "palangres" or "espineles", could produce a 300-400 fish catch only in one night (Matte 1981). A catch of this size ought not to be surprising when we consider historical references such as that describing the visit of French explorer Louis Feuillée to Cobija in 1710. According to the record, Feuillée (1714) purchased between $225-275 \mathrm{~kg}$ of dried fish from local fisherman. Just as an approximate and theoretical exercise, if we consider that drying of fish reduces its weight by roughly $80 \%$ (as a consequence of gutting and dehydration; Burgess et al. 1971), the initial catch weight that Feuillée's purchase represented would likely have been at least five times greater than the dry weight he reported, between $1130-1360 \mathrm{~kg}$. If we use the jack mackerel (Trachurus murphyi Nichols, 1920) as a tentative fish example - one of the most popular and common fish captured in this coast-, it would correspond to 735-906 complete adult individuals (Yańez 1955; Rosario 1970; Vargas et al. 1993).
For determining the species consumed, coastal refuse middens provide the best evidence ${ }^{2}$. The domestic midden at Abtao $5^{3}$, for example, yielded no fewer than 20 fish species, $49.2 \%$ of which were from pelagic habitats and $50.8 \%$ from benthic-demersal settings (Bravo 1981, 1985; Llagostera 1990). Similarly, in Taltal, in a grouping of eleven sites attributed to the Formative period, fourteen fish species were identified (Castelleti 2007; Castelleti \& Maltrain 2010). The diversity of these assemblages exemplifies the multiplicity of strategies and technologies used to catch fish and the wide variety of species preferred by these coastal groups.

\section{FISH ALONG CIRCULATION ROUTES}

No fewer than five Formative period routes connecting the coast with the Atacama Desert interior have been documented in our study area, linking Quillagua, the middle Loa, and Guatacondo (Figs $1 ; 2 ; 4)$. Eleven sites associated with the routes passing through Calate, El Toco and Talabre have been studied from a zooarchaeological perspective (Table 1). These sites appear to be transit stops, composed of a variable number of circular drystone structures and featuring a low density of material culture. These sites possess limited faunal assemblages, with notably more fish than other common taxa such as camelids or rodents (Table 1). Generally, we observed the presence of a varied fish species list, although the majority of these were represented by just a few bony elements. The exceptions included the jack mackerel, corvina drum (Cilus gilberti (Abbott, 1899)), anchovy (Engraulis ringens Jenyns, 1842) and menhaden (Ethmidium maculatum (Valenciennes, 1847)), which were found in the greatest abundance and/or in the majority of sites (Table 1).

Archeological investigation of sites associated with preHispanic transit routes in the area have shown that coastal groups participated actively in circulating their surplus of fish in the desert interior (Cases et al. 2008; Pimentel et al. 2010, 2011, 2017). On their travels, these individuals preferentially consumed fish instead of the resources they would have eventually obtained via exchange with inhabitants of the inland oases. A study of the stomach contents of a coastal individual who died on the El Toco route found fish remains exclusively and the isotopic history of this individual suggested a diet rich in marine resources (Fig. 5); even more, a little textile bag founded in direct association to the individual contained a cactus spine fishhook, another prove of his littoral origin (Cases et al. 2008; Knudson et al. 2012; Pestle et al. 2015b). Equally, in one of the many tombs associated with the ancient route that connected the Loa River mouth with the locality of Calate (25 km from the coast) (Figs 1; 2), a 4-6 year old child was found with a rich array of offerings that included at least three large dried, gutted, headless, skinned fish with caudal fins and vertebrae attached, along with a large number

2. Unfortunately, there are very few systematically excavated and chronologically controlled Formative sites, and as a consequence we can only make a general, preliminary assessment of the diversity of species consumed.

3. Even though this is one of the best-documented coastal sites, it still suffers from a lack of chronological control, making it very difficult to separate the distinct occupational and cultural layers and events. The only date obtained thus far, from the lower strata, is 398-190 cal B.C. (Bravo 1981, 1985; Llagostera 1990). 


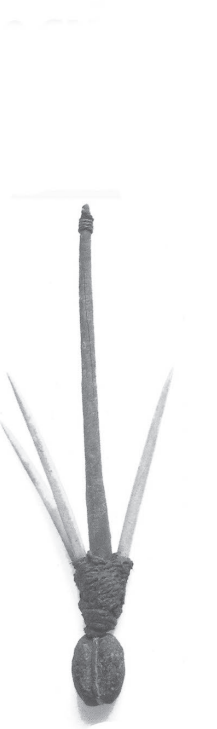

A

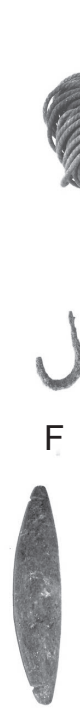

B

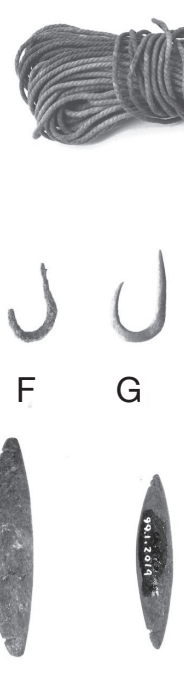

C
K

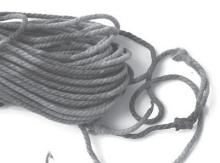

$\circlearrowleft \cup 0$

H I J

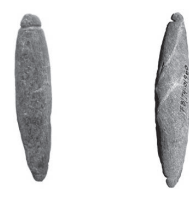

D

E

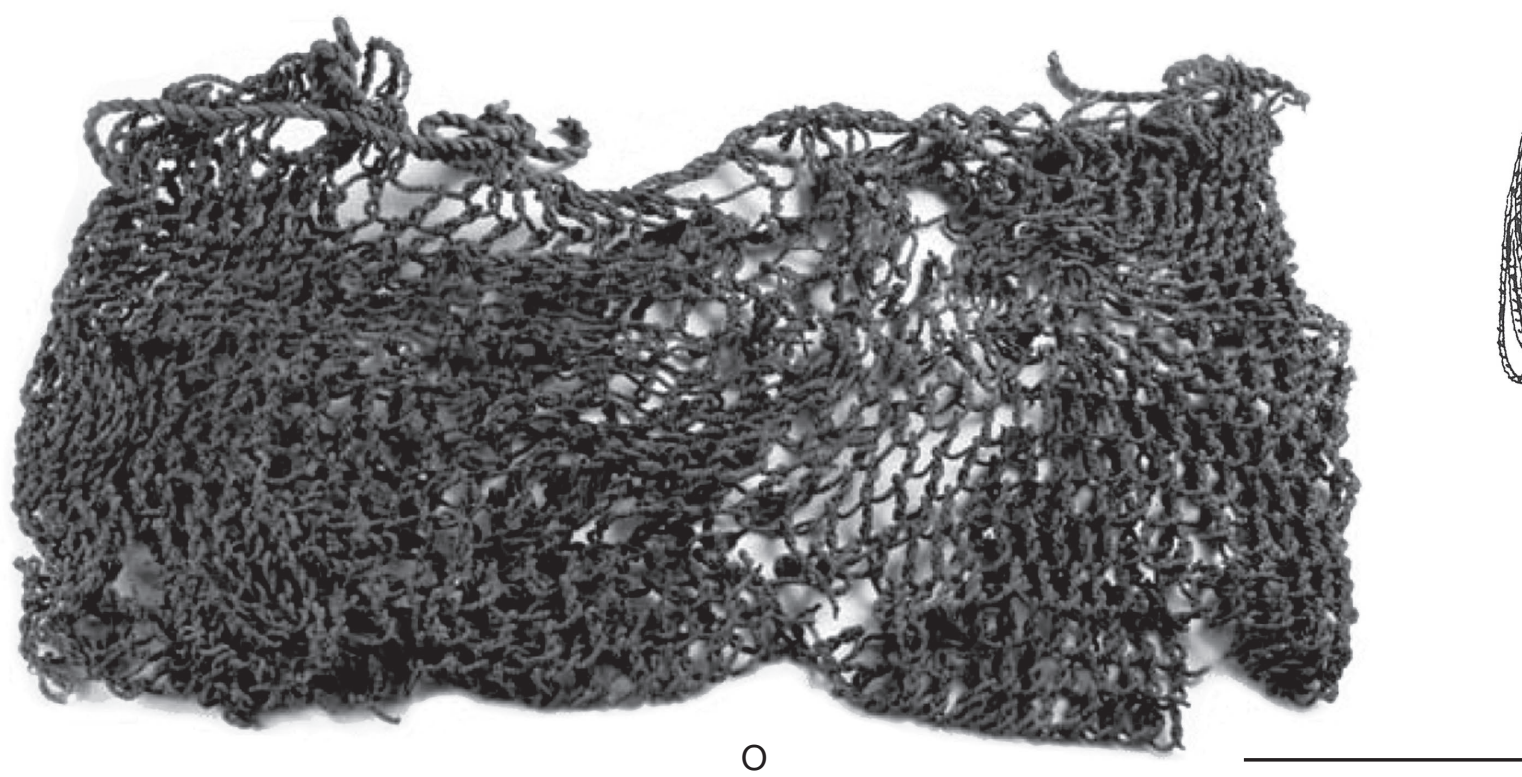

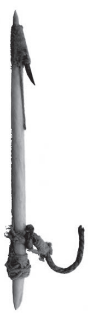

L

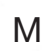

M

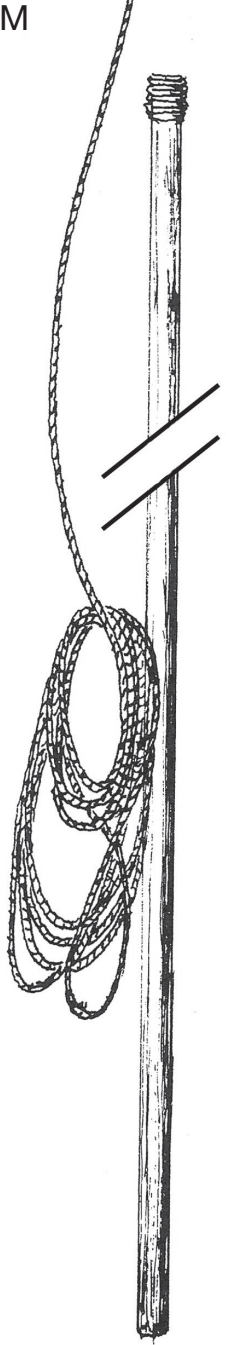

N

FIG. 3. - Coastal fishing toolkit: A, gaff or "garabato" (uncertain chronology; Berenguer 2008: 25); B, C, stone line-weights from Caleta Huelén 20; D, stone line-weight from Hornitos 01; E, stone line-weight from Punta Blanca 01 (the four from the Formative Period; Ballester \& Clarot 2014: 58); F, copper hook from Punta Grande 02; G, bone hook from Michilla 11; H-J, spine cactus hooks from Las Loberas (the five from the Formative Period; Ballester \& Clarot 2014: 58); K, braided cotton line from Las Loberas (Formative Period; Ballester \& Clarot 2014: 58); L, harpoon head made by a bone stem and cactus spine barbs from Caleta Huelén 31 (Formative Period; Spahni 1967: PI. V, 17); M, harpoon head made by a bone stem and cactus spine barbs from Las Loberas (Formative Period; Ballester \& Clarot 2014: 60); N, complete harpoon device scheme (Llagostera 1989: 62, fig. 2); O, complete fishing net from Las Loberas (Formative Period; Sinclaire 2008: 82). Scale bar: $10 \mathrm{~cm}$.

of small unprocessed anchovies (Fig. 6; Torres-Rouff et al. 2012b; Pimentel \& Ugarte 2017; Pimentel et al. 2017). This context has been dated to the late Formative period (Fig. 4), and the child's elevated $\delta 15 \mathrm{~N}$ values indicate a diet based on marine resources (Fig. 5; Torres-Rouff et al. 2012b; Pestle et al. 2015a, b; Pimentel \& Ugarte 2017; Pimentel et al. 2017).

\section{QUILLAGUA, A MEETING PLACE BETWEEN THE COAST} AND THE DESERT

The oasis of Quillagua, $60 \mathrm{~km}$ from the Pacific coast, was one of the main points of entry for fish into the interior (Figs 1;2). During the Formative period the locality was inhabited by both locally-derived groups and others strongly linked to the 


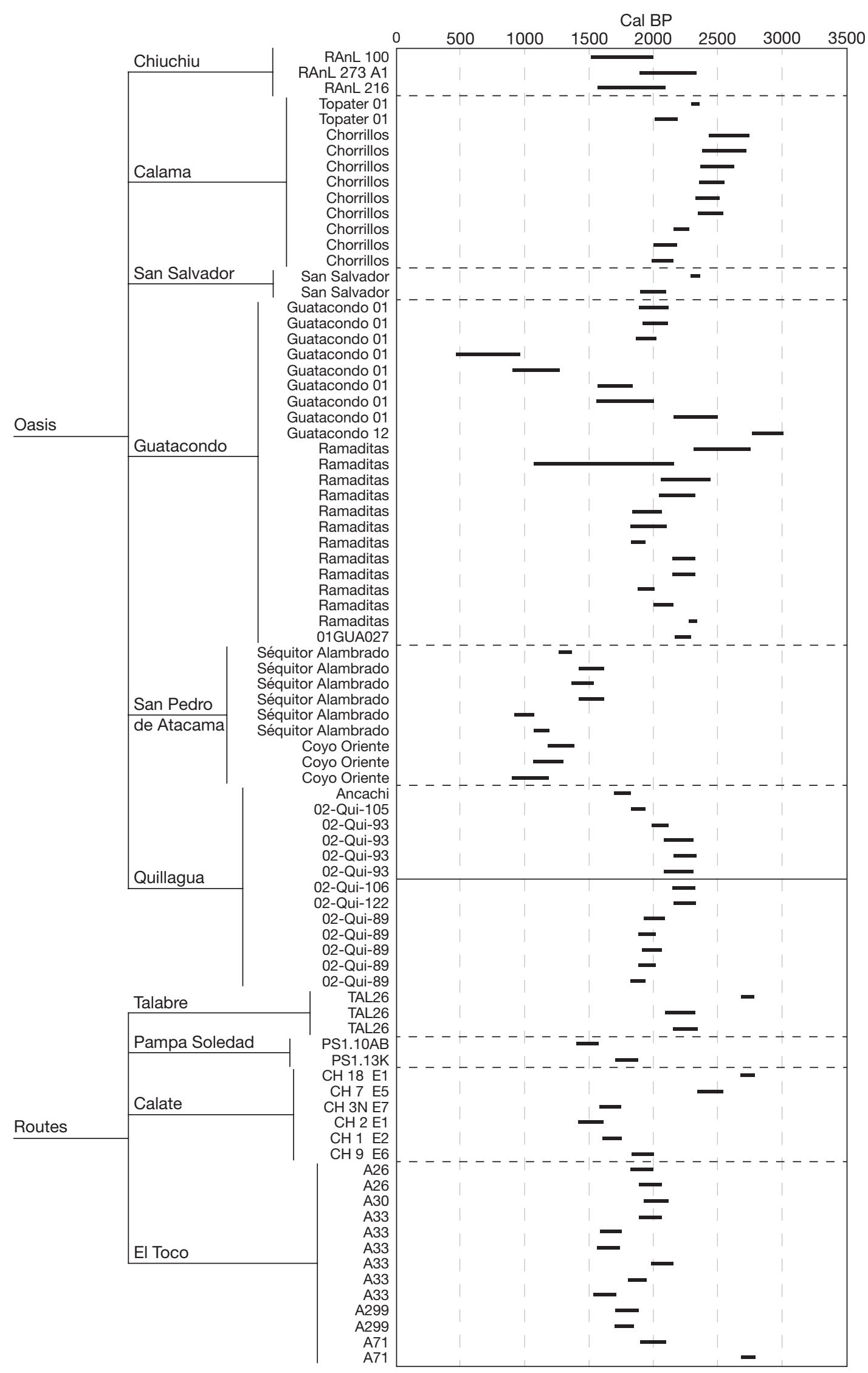

FIG. 4. - Radiocarbon datations from the Formative sites of the Atacama Desert considered in this study. Marine and human material samples were excluded. Calibration made by SHCAL13 curve (Hogg et al. 2013) using Calib 704 (Stuiver et al. 2005). 
TABLE 1. - Fish remains (NISP) from route sites of the Atacama Desert. All sites are referenced in this article, except the funerary site Toco A299, referenced in Cases et al. 2008. Abbreviations: C, cranial; D, domestic; F, funerary; P, postcranial; R, route.

\begin{tabular}{|c|c|c|c|c|c|c|c|c|c|c|c|c|c|}
\hline \multirow[b]{4}{*}{ Order/Species } & \multirow{4}{*}{$\begin{array}{c}\text { Skeletal } \\
\text { part }\end{array}$} & \multicolumn{11}{|c|}{ Locality, site and context } & \multirow[b]{4}{*}{ Total } \\
\hline & & \multicolumn{6}{|c|}{ Ch-Calate } & \multicolumn{4}{|c|}{ El Toco } & \multirow{2}{*}{$\frac{\text { Talabre }}{\text { Tal-26 }}$} & \\
\hline & & Ch 01 & Ch 02 & Ch 03 & Ch 07 & Ch 09 & Ch 18 & Toco A26 & 6 Toco A33 & Toco A71 & Toco A299 & & \\
\hline & & $\mathrm{R}$ & $\mathrm{R}$ & $\mathrm{R}-\mathrm{F}$ & & $\mathrm{R}$ & $\mathrm{R}$ & $\mathrm{R}$ & $\mathrm{R}$ & $\mathrm{R}$ & $\mathrm{F}$ & $\mathrm{R}$ & \\
\hline \multicolumn{14}{|l|}{ Perciformes } \\
\hline \multirow{2}{*}{$\begin{array}{l}\text { Acanthistius pictus } \\
\text { (Tschudi, 1846) }\end{array}$} & $\mathrm{C}$ & - & - & - & 1 & - & - & - & - & - & - & - & 1 \\
\hline & $\mathrm{P}$ & - & - & - & - & - & - & - & - & - & - & - & 0 \\
\hline \multirow{2}{*}{$\begin{array}{l}\text { Aplodactylus punctatus } \\
\text { Valenciennes, } 1832\end{array}$} & $\mathrm{C}$ & - & - & - & - & 1 & - & - & - & - & - & - & 1 \\
\hline & $\mathrm{P}$ & _- & _- & _- & _- & - & - & - & - & _- & - & - & 0 \\
\hline \multirow{2}{*}{ Aucheniounchus sp. } & $\mathrm{C}$ & _- & _- & _- & _- & 1 & - & - & _- & _- & _- & _- & 1 \\
\hline & $\mathrm{P}$ & - & - & - & - & - & - & - & - & - & - & - & 0 \\
\hline \multirow{2}{*}{$\begin{array}{l}\text { Cheilodactylus variegatus } \\
\text { Valenciennes, } 1833\end{array}$} & $\mathrm{C}$ & - & - & - & - & - & - & - & - & - & - & - & 0 \\
\hline & $\mathrm{P}$ & - & 1 & 3 & - & - & - & - & 4 & - & - & 1 & 9 \\
\hline \multirow[t]{2}{*}{ Cilus gilberti (Abbott, 1899) } & $\mathrm{C}$ & - & 7 & 2 & - & - & - & - & - & - & - & - & 9 \\
\hline & $\mathrm{P}$ & - & 11 & 25 & - & 2 & - & - & - & - & - & 2 & 40 \\
\hline \multirow{2}{*}{ Graus nigra Philippi, 1887} & C & - & 1 & - & - & - & - & - & - & - & - & - & 1 \\
\hline & $\mathrm{P}$ & - & 2 & 2 & - & - & - & 1 & - & - & - & 3 & 8 \\
\hline \multirow{2}{*}{$\begin{array}{l}\text { Hemilutjanus macroph- } \\
\text { thalmos (Tschudi, 1846) }\end{array}$} & $\mathrm{C}$ & - & - & - & - & - & - & - & - & - & - & - & 0 \\
\hline & $\mathrm{P}$ & - & 7 & - & - & - & - & - & - & - & - & - & 7 \\
\hline \multirow{2}{*}{$\begin{array}{l}\text { Isacia conceptionis (Cuvier, } \\
\text { 1830) }\end{array}$} & $\mathrm{C}$ & - & - & 1 & - & - & - & - & - & - & - & - & 1 \\
\hline & $\mathrm{P}$ & - & - & - & - & - & - & - & - & _- & - & _- & 0 \\
\hline \multirow[t]{2}{*}{ Labrisomidae } & $\mathrm{C}$ & - & 1 & 1 & - & - & - & - & - & - & 3 & - & 5 \\
\hline & $\mathrm{P}$ & - & - & - & - & - & - & - & 1 & - & 32 & - & 33 \\
\hline Pinguipes chilensis & $\mathrm{C}$ & - & - & 1 & - & - & - & - & - & - & - & - & 1 \\
\hline Valenciennes, 1833 & & & & & & & & & & & & & 2 \\
\hline Prolatilus jugularis & $\begin{array}{l}P \\
C\end{array}$ & $\begin{array}{l}- \\
-\end{array}$ & - & -2 & - & $\begin{array}{l}- \\
-\end{array}$ & - & $\begin{array}{l}- \\
-\end{array}$ & $\begin{array}{l}- \\
-\end{array}$ & $\begin{array}{l}- \\
-\end{array}$ & $\begin{array}{l}- \\
-\end{array}$ & $\begin{array}{l}- \\
-\end{array}$ & $\begin{array}{l}2 \\
0\end{array}$ \\
\hline Valenciennes, 1833 & & & & & & & & & & & & & \\
\hline & $\mathrm{P}$ & - & - & - & - & - & - & - & 1 & - & - & - & 1 \\
\hline Sarda chiliensis (Cuvier, & $\mathrm{C}$ & - & - & - & - & - & - & - & - & - & - & - & 0 \\
\hline & $\mathrm{P}$ & - & 25 & 2 & - & - & - & - & - & - & - & - & 27 \\
\hline Semicossyphus darwini & $\mathrm{C}$ & - & 2 & 4 & - & - & - & - & - & - & - & - & 6 \\
\hline & $P$ & - & 3 & 5 & - & - & - & - & - & - & - & 1 & 9 \\
\hline Trachurus murphyi Nichols, & $\mathrm{C}$ & - & 3 & 4 & - & - & - & 1 & - & - & - & - & 8 \\
\hline & $\mathrm{P}$ & 1 & 64 & 124 & 7 & 65 & - & 8 & 9 & 3 & - & 10 & 291 \\
\hline Chimaeriformes & & & & & & & & & & & & & \\
\hline Callorhinchus callorynchus & C & - & - & - & - & - & - & - & - & - & - & - & 0 \\
\hline & $\mathrm{P}$ & - & - & 1 & - & - & - & - & - & - & - & - & 1 \\
\hline Clupeiformes & & & & & & & & & & & & & \\
\hline Engraulis ringens (Jenyns, & $\mathrm{C}$ & - & - & 60 & - & - & - & - & - & - & - & - & 60 \\
\hline & $\mathrm{P}$ & _- & _- & 199 & - & - & - & - & - & - & - & - & 199 \\
\hline Ethmidium maculatum & $\mathrm{C}$ & - & - & - & - & - & - & - & - & - & - & - & 0 \\
\hline & $\mathrm{P}$ & - & 74 & 17 & - & 20 & - & - & 2 & - & - & - & 113 \\
\hline Pleuronectiformes & & & & & & & & & & & & & \\
\hline Paralichthys microps & $\mathrm{C}$ & - & 3 & 1 & - & 1 & - & - & - & - & - & - & 5 \\
\hline & $\mathrm{P}$ & - & 11 & 6 & - & - & - & - & - & - & - & 8 & 25 \\
\hline Scorpaeniformes & & & & & & & & & & & & & \\
\hline Sebastes oculatus & C & 2 & 1 & - & - & - & - & - & - & - & - & - & 3 \\
\hline & $\mathrm{P}$ & - & 2 & - & - & - & - & - & 2 & - & - & - & 4 \\
\hline Actinopterygii indeterminate & C & - & 22 & 132 & - & $\overline{166}$ & 1 & - & 9 & - & - & $\begin{array}{r}1 \\
110\end{array}$ & $\begin{array}{r}165 \\
1696\end{array}$ \\
\hline & $\mathrm{P}$ & 4 & 650 & 533 & 9 & 166 & 80 & 13 & 103 & 28 & - & 110 & 1696 \\
\hline Total & & 7 & 890 & 1125 & 17 & 256 & 81 & 23 & 131 & 31 & 35 & 136 & 2732 \\
\hline
\end{tabular}




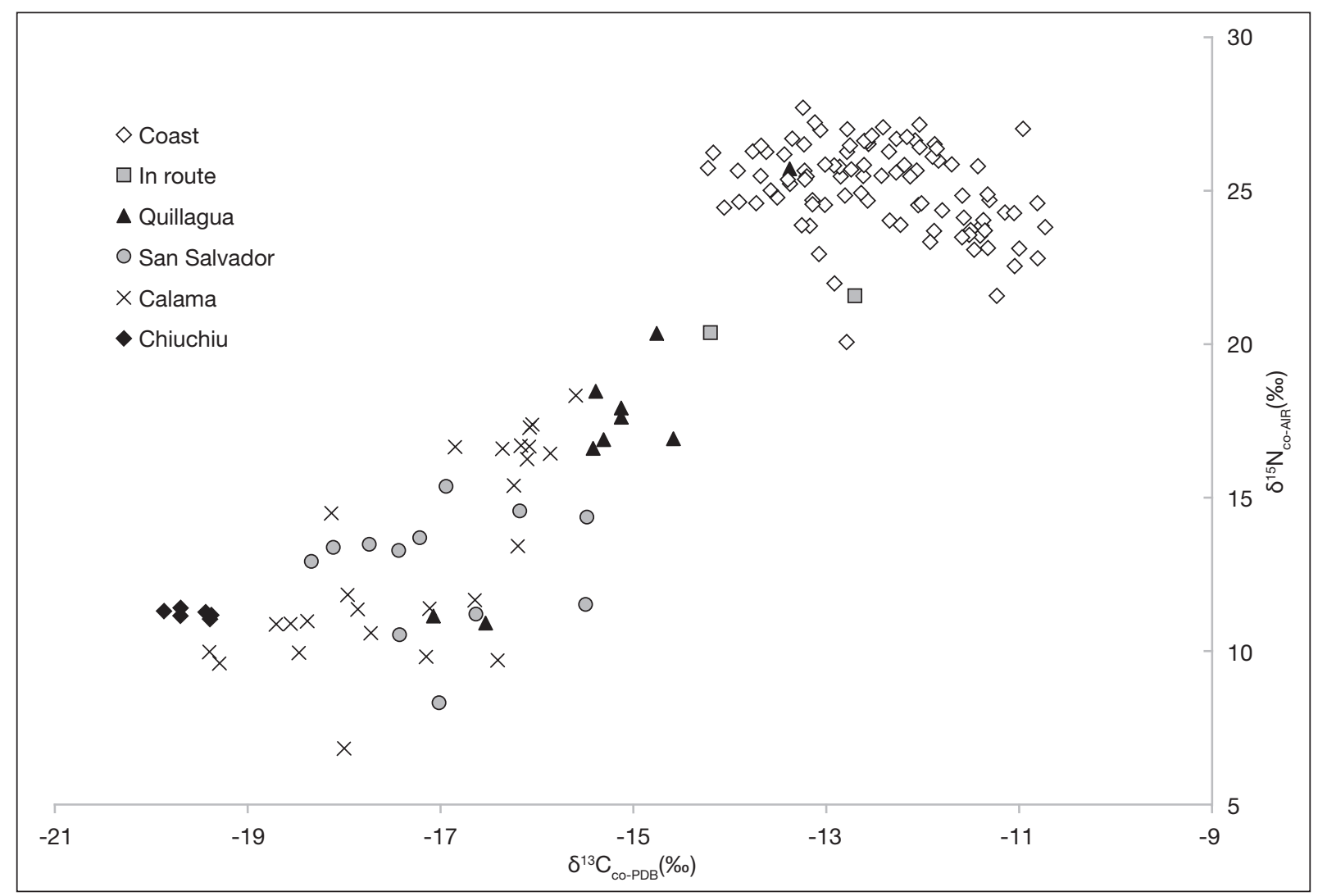

FIG. 5. - Human isotope values from the different cemeteries mentioned, separated by localities (Knudson et al. 2012; Torres-Rouff et al. 2012a; Ballester \& Clarot 2014; Pestle et al. 2015a, b; Pestle 2017).

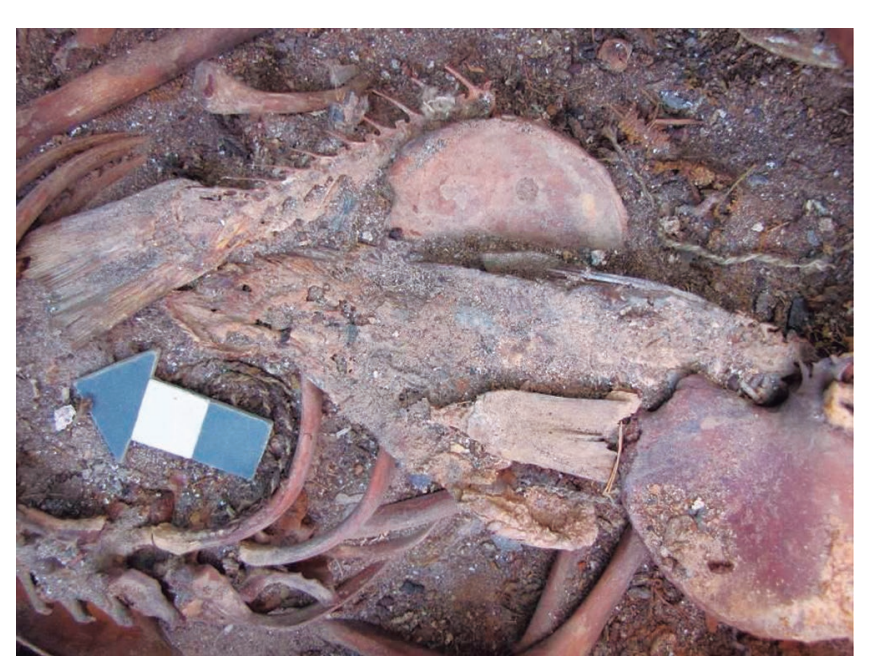

Fig. 6. - Headless dried fish offered to a 4-6 years old infant in the route of Calate-Loa River mouth.

coastal world, and as such everyday life would have been shared by people who were geographically and culturally distinct (Gallardo et al. 2017a). The presence of a non-local contingent in Quillagua has been inferred, in part, from the $\delta 15 \mathrm{~N}$ signatures of individuals interred in the cemetery of Ancachi (02-Qui-75), situated $6 \mathrm{~km}$ downriver from Quillagua proper (Latcham 1938; Gallardo et al. 1993a, b; Pestle 2017). These signatures correspond to a diet rich in marine protein, in some cases with values equivalent to those seen in coastal peoples (Fig. 5; Pestle et al. 2015a; Pestle 2017). This cemetery, along with two others located upriver, follow the same architectural and funerary patterns than coastal cemeteries - as tumuli fields. These findings attest to an intimate connection and close cultural linkage between at least some of the inhabitants of Quillagua and the coastal world (Latcham 1938; Spahni 1967; Núñez 1971; Ballester \& Clarot 2014; Gallardo et al. 2017a). Data from residential middens in Ancachi confirm the conclusions derived from stable isotope analysis.

Also in the Ancachi area (Fig. 1), the excavation of three large Formative period residential camps (02-Qui-122, 02-Qui-115 \& 02-Qui-106) yielded a great quantity of cranial and postcranial bones from diverse marine fish species (Table 2; Gallardo et al. 1993a, b, 2017a). This zooarchaeological evidence reinforce the interpretation of a high marine consumption in the neighbour cemetery. Upriver and near the actual town of Quillagua, in 02-Qui-89 (Agüero et al. 2001, 2006), an artificial mound associated with a cluster of circular structures with central patios dated to the Formative period (Fig. 4), the bones, skin, and scales of Pacific fish have been recently recovered and identified (Table 2). Excavations performed at the site also yielded human coprolites containing fish vertebrae, demonstrating the human consumption of this resource. Additionally, excavations at a similar residential settlement called 02-Qui-93 yielded more than 770 fish bones representing at least 10 different species (Table 2). 
TABLE 2. - Fish remains (number of identified specimens) from Quillagua sites.

\begin{tabular}{|c|c|c|c|c|c|c|c|c|}
\hline \multirow[b]{2}{*}{ Order/Species } & \multirow[b]{2}{*}{ Skeletal part } & \multicolumn{6}{|c|}{ Locality, site and context } & \multirow[b]{2}{*}{ Total } \\
\hline & & $\begin{array}{l}\text { Quillagua } \\
\text { 02-Qui-89 } \\
\text { Domestic }\end{array}$ & $\begin{array}{c}\text { Quillagua } \\
\text { 02-Qui-93 } \\
\text { Domestic }\end{array}$ & $\begin{array}{c}\text { Quillagua } \\
02-Q u i-105 \\
\text { Funerary }\end{array}$ & $\begin{array}{c}\text { Quillagua } \\
502-Q u i-122 \\
\text { Domestic }\end{array}$ & $\begin{array}{c}\text { Quillagua } \\
\text { 02-Qui-115 } \\
\text { Domestic }\end{array}$ & $\begin{array}{c}\text { Quillagua } \\
502-Q u i-106 \\
\text { Domestic }\end{array}$ & \\
\hline \multicolumn{9}{|l|}{ Perciformes } \\
\hline \multirow{2}{*}{ Acanthistius pictus (Tschudi, 1846) } & Cranial & - & - & - & - & - & 1 & 1 \\
\hline & Postcranial & - & - & - & - & 3 & - & 3 \\
\hline \multirow{2}{*}{ Cheilodactylus variegatus Valenciennes, 1833} & Cranial & - & - & - & - & 1 & - & 1 \\
\hline & Postcranial & 28 & 64 & - & - & 1 & 3 & 96 \\
\hline \multirow[t]{2}{*}{ Cilus gilberti (Abbott, 1899) } & Cranial & - & - & - & 1 & 1 & - & 2 \\
\hline & Postcranial & 10 & 28 & - & 1 & 1 & 3 & 43 \\
\hline \multirow[t]{2}{*}{ Graus nigra Philippi, 1887} & Cranial & - & - & - & 1 & - & - & 1 \\
\hline & Postcranial & - & 1 & - & - & - & - & 1 \\
\hline \multirow{2}{*}{ Hemilutjanus macrophthalmos (Tschudi, 1846) } & Cranial & - & - & - & - & - & - & 0 \\
\hline & Postcranial & - & 2 & - & - & - & - & 2 \\
\hline \multirow[t]{2}{*}{ Paralabrax humeralis (Valenciennes, 1828) } & Cranial & - & - & - & - & - & - & 0 \\
\hline & Postcranial & 2 & 1 & - & - & 19 & - & 22 \\
\hline \multirow[t]{2}{*}{ Pinguipedidae } & Cranial & - & - & - & - & - & - & 0 \\
\hline & Postcranial & - & 10 & - & - & - & - & 10 \\
\hline \multirow[t]{2}{*}{ Sarda chiliensis (Cuvier, 1832) } & Cranial & - & - & - & - & - & - & 0 \\
\hline & Postcranial & 2 & 26 & - & 9 & 13 & 1 & 51 \\
\hline \multirow[t]{2}{*}{ Sciaena deliciosa (Tschudi, 1846) } & Cranial & - & - & - & - & - & 1 & 1 \\
\hline & Postcranial & - & - & - & - & 4 & - & 4 \\
\hline \multirow[t]{2}{*}{ Semicossyphus darwini (Jenyns, 1842) } & Cranial & - & - & - & 4 & 7 & - & 11 \\
\hline & Postcranial & - & 3 & - & - & - & - & 3 \\
\hline \multirow[t]{2}{*}{ Seriolella violacea Guichenot, 1848} & Cranial & - & - & - & - & - & - & 0 \\
\hline & Postcranial & - & - & - & - & 2 & 2 & 4 \\
\hline \multirow[t]{2}{*}{ Trachurus murphyi Nichols, 1920} & Cranial & - & - & - & - & 1 & - & 1 \\
\hline & Postcranial & 96 & 72 & - & 1 & 42 & 7 & 218 \\
\hline \multirow[t]{2}{*}{ Xiphias gladius Linnaeus, 1758} & Cranial & - & - & - & - & - & - & 0 \\
\hline & Postcranial & 1 & - & - & - & - & - & 1 \\
\hline \multicolumn{9}{|l|}{ Pleuronectiformes } \\
\hline Paralichthys microps (Günther, 1881) & Cranial & - & 1 & 1 & 1 & - & 1 & 4 \\
\hline & Postcranial & - & - & - & - & 1 & - & 1 \\
\hline Paralichthyidae indet. & Cranial & - & - & - & - & - & - & 0 \\
\hline & Postcranial & 11 & 155 & - & 9 & 18 & 13 & 206 \\
\hline Ophidiiformes & & & & & & & & \\
\hline Genypterus sp. & Cranial & - & - & - & 1 & - & - & 1 \\
\hline & Postcranial & - & - & - & - & - & - & 0 \\
\hline Scorpaeniformes & & & & & & & & \\
\hline Sebastes oculatus Valenciennes, 1833 & Cranial & - & - & - & - & - & - & 0 \\
\hline & Postcranial & 3 & - & - & - & - & - & 3 \\
\hline Clupleiformes & & & & & & & & \\
\hline Clupeiforme indet. & Cranial & - & - & - & - & - & - & 0 \\
\hline & Postcranial & 5 & - & - & - & - & - & 5 \\
\hline Lamniformes & & & & & & & & \\
\hline Isurus oxyrinchus Rafinesque, 1810 & Cranial & - & - & - & - & 1 & - & 1 \\
\hline & Postcranial & - & - & - & - & - & - & 0 \\
\hline Squalimorpha indet. & Cranial & _- & _- & - & _- & 1 & - & 1 \\
\hline & Postcranial & - & - & - & - & 1 & - & 1 \\
\hline Actinopterygii indet. & Cranial & - & - & - & 1 & 6 & 6 & 13 \\
\hline & Postcranial & 81 & 408 & - & 54 & 89 & 64 & 696 \\
\hline Total & & 239 & 771 & 1 & 83 & 212 & 102 & 1408 \\
\hline
\end{tabular}

Direct routes between Quillagua and the coast were used intensively during the Formative, both those stretching to Tocopilla and those that paralleled the Loa River down to the sea (Fig. 1). The latter passed through the area of Calate, where fish remains have commonly been found both at rest stops and in the tombs of deceased travelers (Table 1; Torres-Rouff et al. 2012b; Pimentel \& Ugarte 2017; Pimentel et al. 2017).

The traditions of shared occupation of the valley and frequent consumption of products from the Pacific coast were maintained in the centuries following the Formative period, establishing greater historical continuity and strengthening social bonds between Quillagua's inhabitants and those living by the sea (Santana 2011; Santana et al. 2015). Without a doubt, the richness of the ecosystem of the valley and the relative proximity of the coast (compared to the remoteness of other oases) helped transform the area into a space of encounter and a hub for people living along the Pacific coast, and this strengthened both economic relations and cultural ties. 
THE CONSUMPTION OF FISH IN GUATACONDO, the Middle Loa and the Atacama Salt Flat

During the Formative period, fish circulated far inland to major population centers, including the oases of Calama and Chiuchiu (c. 140 and $160 \mathrm{~km}$ from the coast, respectively), the ravine confluences of the Pampa del Tamarugal, such as Guatacondo and Mani (c. $90 \mathrm{~km}$ from the coast), and the oases around the Salar de Atacama, such as San Pedro (c. $210 \mathrm{~km}$ from the coast) and Toconao (c. $240 \mathrm{~km}$ from the coast) (Figs 1; 2; Pollard 1971; Benavente 1978, 1981, 1998; Follet 1980; Orellana 1991; Cartajena 1994; Thomas et al. 1994; Rivera et al. 1996; González \& Westfall 2010; Agüero \& Uribe 2011).

At the Guatacondo ravine, the Ramaditas and Guatacondo 01 villages were probably the main residential hubs during the Formative period (Fig. 4; Mostny \& Niemeyer 1963; Mostny 1970a, b; Meighan \& True 1980; Rivera et al. 1996; Rivera 2002; Urbina et al. 2012; Adán et al. 2013). Notable among the mass of domestic refuse recovered from the excavations of these occupied areas was the presence of vertebral bones from at least seven fish species, whereas fish cranial bones were entirely absent (Table 3). Recent excavations of a waste mound in the village of Ramaditas corroborated the above findings (Table 3) and provided a wider variety of species than previously recorded (Follet 1980; Rivera et al. 1996). Not far from the main Guatacondo 01 site, in the Formative residential complex of 01 GUA027 (Fig. 4), the remains of at least nine different fish species were recovered, all from them postcranial skeleton (Table 3).

Human coprolites recovered from the residential areas of Guatacondo 01 and Ramaditas have also confirmed the local consumption of fish. At Guatacondo 01, fish vertebrae were identified in one of the sixteen human feces collected as sample (Williams 1980), while at Ramaditas fish consumption can be inferred from the results of two related findings. One came from the analysis of protein residues using antiserum, which yielded a positive result for the presence of anchoveta (Scott et al. 2005); while the other was the presence of roughly 200 eggs of the parasite Diphyllobothrium spp. in four fecal samples from Area 1 of the village (Rivera 2002). This parasite, very common among the coastal people of northern Chile and southern Peru, uses fish as its host during maturation and is transmitted to humans as a result of the consumption of raw, dried, smoked, or poorly cooked fish (Callen $\&$ Cameron 1960; Ferreira et al. 1984; Reinhard 1992; Arriaza 1995; Reinhard \& Urban 2003; Carvalho et al. 2003; Santoro et al. 2003; Araújo et al. 2011).

The middle course of the Loa River contains a series of stable residential sites and dense cemeteries dating to the Formative period (Fig. 4). Some $80 \mathrm{~km}$ from the coast and $40 \mathrm{~km}$ west of Calama (Figs 1;2), on the south bank of the San Salvador river, a village has been found, consisting of 10 to 15 semisubterranean structures now filled in with aeolian soil. These residences are associated with a looted cemetery of between 20 and 25 graves, and calibrated radiocarbon dates obtained for both residential and mortuary contexts situates them in the Middle Formative period (Fig. 4; Torres-Rouff et al. 2012a).
Excavation of one residential precinct yielded a considerable volume of food remains, almost all of which consisted of algarrobo (Prosopis sp.) fruit and fish vertebrae. More than 2000 fish bone fragments were recovered, all vertebral bodies, spines, and arches with not a single cranial fragment among them (Table 3). As the absence of cranial fragments hindered taxonomic identification, four species could be identified from the large assemblage recovered (Table 3; Castillo 2011, 2015; Castillo et al. 2017).

This appreciable volume of fish remains so far from the sea makes sense when one takes into account the $\delta 15 \mathrm{~N}$ values of individuals from the cemetery of the site. Of the 15 sampled individuals from San Salvador, at least 12 showed evidence of marine product consumption, averaging approximately between $8 \%$ to $25 \%$ of their overall protein diet (Fig. 5; Torres-Rouff et al. 2012a; Pestle et al. 2015a, b; Pestle 2017).

In the present-day oasis of Calama, the best evidence for Formative fish consumption comes from funerary contexts. In the Regimiento Chorrillos and Topater 01 (Fig. 4) cemeteries coastal goods were frequently provided as funerary offerings, and fish remains were no exception (Table 3; Latcham 1938; Thomas et al. 1994; González \& Westfall 2010). One such offering found at Topater 01 is particularly noteworthy. The item (Fig. 7) is a composite food, a single morsel that included a piece of dried fish filet along with a complete dried anchovy tied together with three rounds of string knotted at the ends. It is a sophisticated serving to be sure, one that combines in a single mouthful, two distinct species of fish and two distinct preparations - one butchered and dressed, the other entire-, transforming the whole into more than simply the sum of its parts to offer much more than simple nutritional value.

Additionally, eggs (40 in total) of the parasite Diphyllobothrium spp. were identified among an assemblage of 179 human fecal samples obtained from six funerary contexts in the Regimiento Chorrillos cemetery, demonstrating that at least some fraction of the deceased people buried in that cemetery had consumed incompletely cooked, raw, or dried fish (Castells et al. 2010).

The $\delta 15 \mathrm{~N}$ values of individuals from the Villa Chuquicamata cemetery, in the same oasis of Calama (Fig. 5), further demonstrates that the ingestion of marine resources was far more than an occasional or sporadic event; all individuals interred therein display some level of consumption of these products and for certain individuals these products accounted for up to $50 \%$ of their diet (Pestle et al. 2015a, b; Pestle 2017). Additionally, the analysis of individuals from the Regimiento Chorrillos and Topater 01 cemeteries (two and 12, respectively) show that one individual from each had $\delta 15 \mathrm{~N}$ isotopic values consistent with a diet that included marine resources (Fig. 5; Pestle et al. 2015a, b; Pestle 2017).

Upriver, at the Chiuchiu oasis, is a complex of domestic settlements and at least one cemetery attributable to the Formative period (Figs $1 ; 2 ; 4)$. At three of the most important residential settlements there-Chiuchiu 200, Chiuchiu Cemetery, and RAnL 100 (Fig. 4) - the frequency of fish remains in waste deposits was low, although no quantitative data or species identification are available for those sites (Pollard 1971; Benavente 1978, 
TABLE 3. - Fish remains (NISP) from interior valley, oases and ravines sites of the Atacama Desert. $\times$, presence of fish remains without possibility of estimating the number of individuals. Abbreviations: D, domestic; F, funerary; Or., Oriente.

\begin{tabular}{|c|c|c|c|c|c|c|c|c|c|c|c|c|c|c|c|c|c|}
\hline \multirow[b]{3}{*}{ Order/Species } & \multirow[b]{3}{*}{ Skeletal part } & \multicolumn{15}{|c|}{ Locality, site, context and reference } & \\
\hline & & \multirow{2}{*}{ 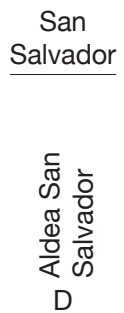 } & \multicolumn{4}{|c|}{ Guatacondo } & \multicolumn{2}{|c|}{ Calama } & \multicolumn{4}{|c|}{ Chiuchiu } & \multicolumn{4}{|c|}{$\begin{array}{c}\text { San Pedro } \\
\text { de Atacama }\end{array}$} & \\
\hline & & & 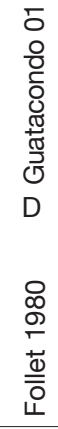 & 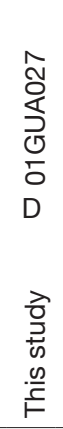 & 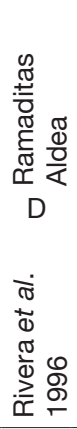 & 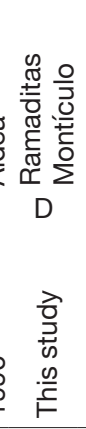 & 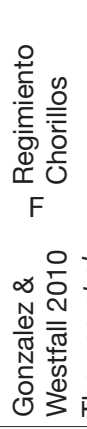 & 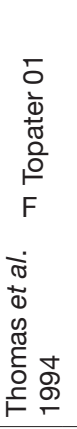 & 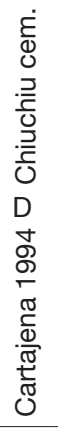 & 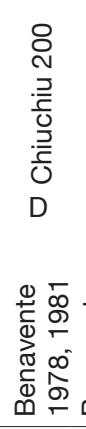 & 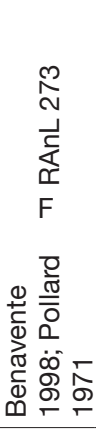 & 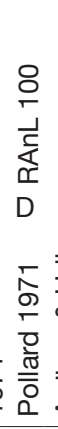 & 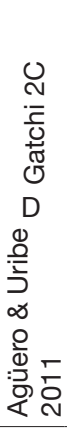 & 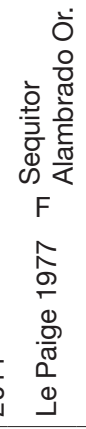 & 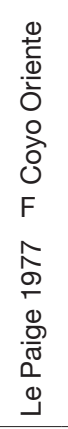 & 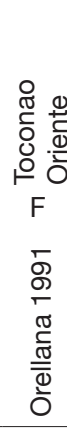 & Total \\
\hline \multicolumn{18}{|l|}{ Perciformes } \\
\hline \multirow[t]{2}{*}{ Cilus gilberti (Abbott, 1899) } & Cranial & - & - & - & - & - & - & - & - & - & - & - & - & - & - & - & 0 \\
\hline & Postcranial & 55 & 3 & 2 & - & 1 & $x$ & - & - & - & - & - & - & - & - & - & 61 \\
\hline \multirow[t]{2}{*}{$\begin{array}{l}\text { Cheilodactylus variegatus } \\
\text { Valenciennes, } 1833\end{array}$} & Cranial & - & - & - & - & - & - & - & - & - & - & - & - & - & - & - & 0 \\
\hline & Postcranial & - & - & 2 & - & 4 & - & - & - & - & - & - & - & - & - & - & 6 \\
\hline \multirow[t]{2}{*}{ Graus nigra Philippi, 1887} & Cranial & - & - & - & - & - & - & - & - & - & - & - & - & - & - & - & 0 \\
\hline & Postcranial & 22 & - & - & - & - & - & - & - & - & - & - & - & - & - & - & 22 \\
\hline \multirow{2}{*}{$\begin{array}{l}\text { Hemilutjanus macrophthalmos } \\
\text { (Tschudi, 1846) }\end{array}$} & Cranial & - & - & - & - & - & - & - & - & - & - & - & - & - & - & - & 0 \\
\hline & Postcranial & - & - & 1 & - & - & - & - & - & - & - & - & - & - & - & - & 1 \\
\hline \multirow[t]{2}{*}{ Sarda chiliensis (Cuvier, 1832) } & Cranial & - & - & - & - & - & - & - & - & - & - & - & - & - & - & - & 0 \\
\hline & Postcranial & - & 13 & - & - & - & - & - & - & - & - & - & - & - & - & - & 13 \\
\hline \multirow[t]{2}{*}{$\begin{array}{l}\text { Semicossyphus darwini } \\
\text { (Jenyns, 1842) }\end{array}$} & Cranial & - & - & - & - & - & - & - & - & - & - & - & - & - & - & - & 0 \\
\hline & Postcranial & - & 2 & 1 & - & - & - & - & - & - & - & - & - & - & - & - & 3 \\
\hline \multirow{2}{*}{ Seriolella violacea Guichenot, 1848} & Cranial & - & - & - & - & - & - & - & - & - & - & - & - & - & - & - & 0 \\
\hline & Postcranial & - & - & 7 & - & 2 & - & - & - & - & - & - & - & - & - & - & 9 \\
\hline \multirow[t]{2}{*}{ Trachurus murphyi Nichols, 1920} & Cranial & - & - & - & - & - & - & - & - & - & - & - & - & - & - & - & 0 \\
\hline & Postcranial & 244 & 6 & 1 & - & - & - & - & - & - & - & - & - & - & - & - & 251 \\
\hline \multicolumn{18}{|l|}{ Clupeiformes } \\
\hline \multirow[t]{2}{*}{ Engraulis ringens Jenyns, 1842} & Cranial & - & - & - & - & - & - & $x$ & - & - & - & - & - & - & - & - & 0 \\
\hline & Postcranial & - & - & - & - & - & - & $x$ & - & - & - & - & - & - & - & - & 0 \\
\hline \multirow[t]{2}{*}{ Clupeiformes indet. } & Cranial & - & - & - & - & - & - & - & - & - & - & - & - & - & - & - & 0 \\
\hline & Postcranial & - & - & 12 & - & - & - & - & - & - & - & - & - & - & - & - & 12 \\
\hline \multicolumn{18}{|l|}{ Ophidiiformes } \\
\hline \multirow[t]{2}{*}{ Genypterus sp. } & Cranial & - & - & - & - & - & - & - & - & - & - & - & - & - & - & - & 0 \\
\hline & Postcranial & 2 & - & - & - & - & - & - & - & - & - & - & - & - & - & - & 2 \\
\hline Paralichthyidae indet. & Cranial & - & - & - & - & - & - & - & - & - & - & - & - & - & - & - & 0 \\
\hline & Postcranial & - & 10 & 30 & - & 9 & - & - & - & - & - & - & - & - & - & - & 49 \\
\hline Scorpaeniformes & & & & & & & & & & & & & & & & & \\
\hline Sebastes oculatus Valenciennes, & Cranial & - & - & - & - & - & - & - & - & - & - & - & - & - & - & - & 0 \\
\hline & Postcranial & - & - & 2 & - & - & - & - & - & - & - & - & - & - & - & - & 2 \\
\hline Indeterminate & & & & & & & & & & & & & & & & & \\
\hline Actinopterygii indet. & Cranial & - & - & 1 & - & - & - & $x$ & - & - & - & - & - & - & - & - & 1 \\
\hline & Postcranial & 1556 & - & 64 & 17 & 36 & $x$ & $x$ & $x$ & $x$ & 2 & 4 & $x$ & 1 & 10 & 1 & 1691 \\
\hline Total & & 1879 & 34 & 123 & 17 & 52 & $x$ & $x$ & $x$ & $x$ & 2 & 4 & $x$ & 1 & 10 & 1 & 2123 \\
\hline
\end{tabular}

1981; Cartajena 1994). Only two fish vertebrae were reported from the cemetery of Chiuchiu 273 (Benavente 1998), and the six human samples analyzed by stable isotopes technique from this cemetery does not show clear evidence of marine proteins consumption (Pestle et al. 2015a, b; Pestle 2017).

Further south, around the Atacama Salt Flat, the frequency of fish remains decreases significantly, but however low that rate is, the mere presence of such remains demonstrates that they were in some degree obtained and consumed. Fish vertebrae have been recovered from both Late Archaic and Formative layers from the Ghatchi $2 \mathrm{C}$ village near the Vilama River (Agüero \& Uribe 2011) and fish have been identified among the funerary offerings of three individuals, each buried in a different cemetery-Séquitor Alambrado, Coyo Oriente, and Toconao Oriente (Table 3; Le Paige 1977; Orellana 1991). 

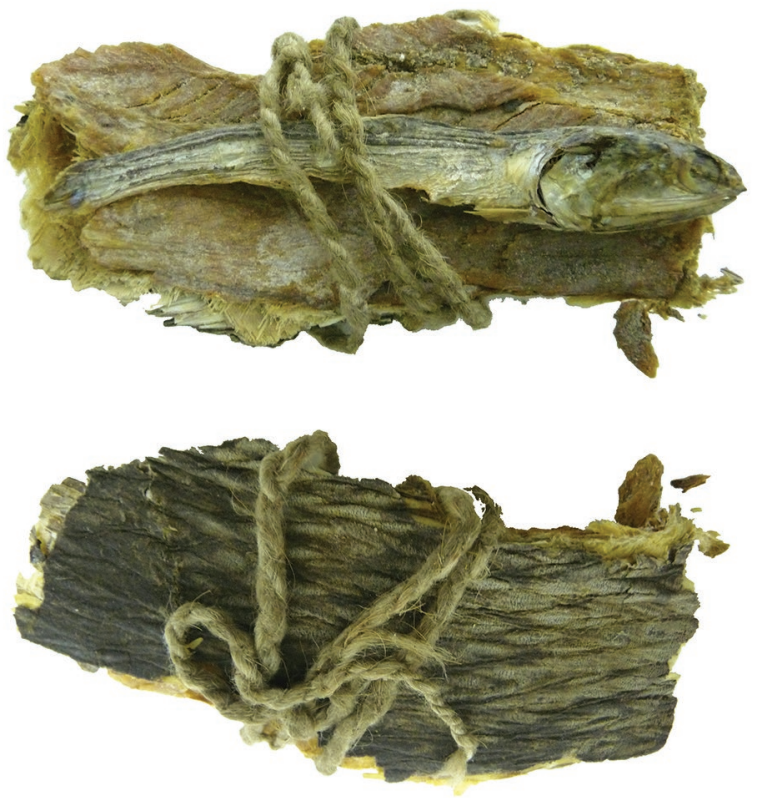

FIG. 7. - Composite food made of two kind of fishes tied together by a braided rope: a complete anchoveta and a boneless fillet of a bigger fish. Topater 01 cemetery (Gallardo 2017: fig. 2). Scale bar: $5 \mathrm{~cm}$.

\section{THE WAYS OF FISH BEYOND THE SEA}

The zooarchaeological, isotopic, and paleofecal evidence shows clearly that people living in the valleys, oases, and ravines of the interior Atacama Desert during the Formative period augmented their diets, to differing degrees, with fish products obtained through exchange from the Pacific Ocean coast (Williams 1980; Rivera 2002; Scott et al. 2005; Castillo 2011, 2015; Santana et al. 2012; Torres-Rouff et al. 2012a; Pestle et al. 2015a, b; Pestle 2017). These products were transported by both coastal and inland agents who traversed great expanses of desert, sustaining themselves by consuming the selfsame fish, the remains of which have been found alongside the routes they traveled (Cases et al. 2008; Pimentel et al. 2010, 2011; Pimentel 2012; Knudson et al. 2012; Torres-Rouff et al. 2012b; Pimentel \& Ugarte 2017).

While residential sites on the coast provide evidence of the intensive consumption of a wide range of marine fish species $(n=30)$ (Bravo 1981, 1985; Llagostera 1990; Castelleti 2007; Castelleti \& Maltrain 2010), along the trade routes and in the interior the intensity of fish consumption decreased as a function of distance from the coast and the variety of species consumed also diminished $(n=19$ in trade routes, $\mathrm{n}=19$ in Quillagua oasis, $\mathrm{n}=11$ in Guatacondo, and $\mathrm{n}=12$ in Loa River Middle and Atacama Salt Flat) (Fig. 8; Table 4). The $\delta 15 \mathrm{~N}$ isotopic values from the coast and the interior localities decrease in the same degree, in an inverse relation to the distance, from a very high consumption of marine proteins along the littoral settlements through an almost nil in Chiuchiu and the Atacama Salt Flat populations (see Pestle et al. 2015a, b; Fig. 3). Probably, in the last places - and more distant from the coast - fish arrived only occasionally and operated primarily as an exotic and scarce good; while in intermediary valleys, as Calama, San Salvador and Guatacondo, fish has functioned at the same time as food and as an exotic good. Indeed, in Quillagua and some coastal desert trails, fish was principally a food resource, just like in the coastal settlements.

Acknowledging the limitations of our analysis and the general lack of in-depth research, it should be noted that the fish species in circulation in the desert interior were, in general, the same ones that were most frequently caught and consumed on the coast (Table 4), although the methods used to catch them included alternately hooks, gaffs, harpoons and nets, performed by costal people.

Among those species, anchovies had to be captured using nets with small openings or may have occasionally been harvested from beachings of large schools. Tracking and catching of anchovies would also have facilitated access to a number of larger fish species, all of which would have been transported after capture. This "cascade" greatly increased the importance of the humble anchovy, even if they were less captured and consumed, probably endowing it with special value in the ichthyological pantheon of the coastal world. Thus, this species was not only an important local food source; it was also (at least partly) responsible for offshore seasonal productivity and thus with the coastal-interior product/commodity exchange network. For example, schooling pelagic species such as the jack mackerel and bonito (Sarda chiliensis chiliensis (Cuvier, 1832)), frequent the coast in great numbers in spring and summer, where they feed on smaller fish such as the anchovy and thereby increase their value as a human food source (Yañez 1955; Rosario 1970; Medina \& Arancibia 2002). A similar situation holds with the flounder (Paralichthyidae), which consumes mainly anchovies and some crustaceans while inhabiting a more circumscribed range of marine environments including sandy, soft-bottomed areas (Silva \& Oliva 2010). In this sense, the anchovy's value was probably not completely lied just to the nutritional importance to humans, but also into its capability to attract and bring to the coast the other fish species.

Another group of fish frequently sought by coastal people, albeit one that does not live in large schools, includes the cusk eel (Genypterus sp.), sea chub (Graus nigra Philippi, 1887), and Peruvian morwong (Cheilodactylus variegatus Valenciennes, 1833), all of which inhabit the rocky and craggy seabottom where they consume mollusks, echinoderms, and crustaceans (Yañez 1955; Fuentes 1981a, b, 1982; Flores \& Rendic 2011). The capture of these species relied heavily on the use of harpoons and hand lines, resulting in the selective capture of single individuals. These species were also transported to the interior where they were consumed by desert residents.

The fish species that we have identified in the interior settlements studied inhabit different ocean zones, including the neritic, oceanic, pelagic, and benthic. The defining characteristic of the overall fish assemblage is the high 


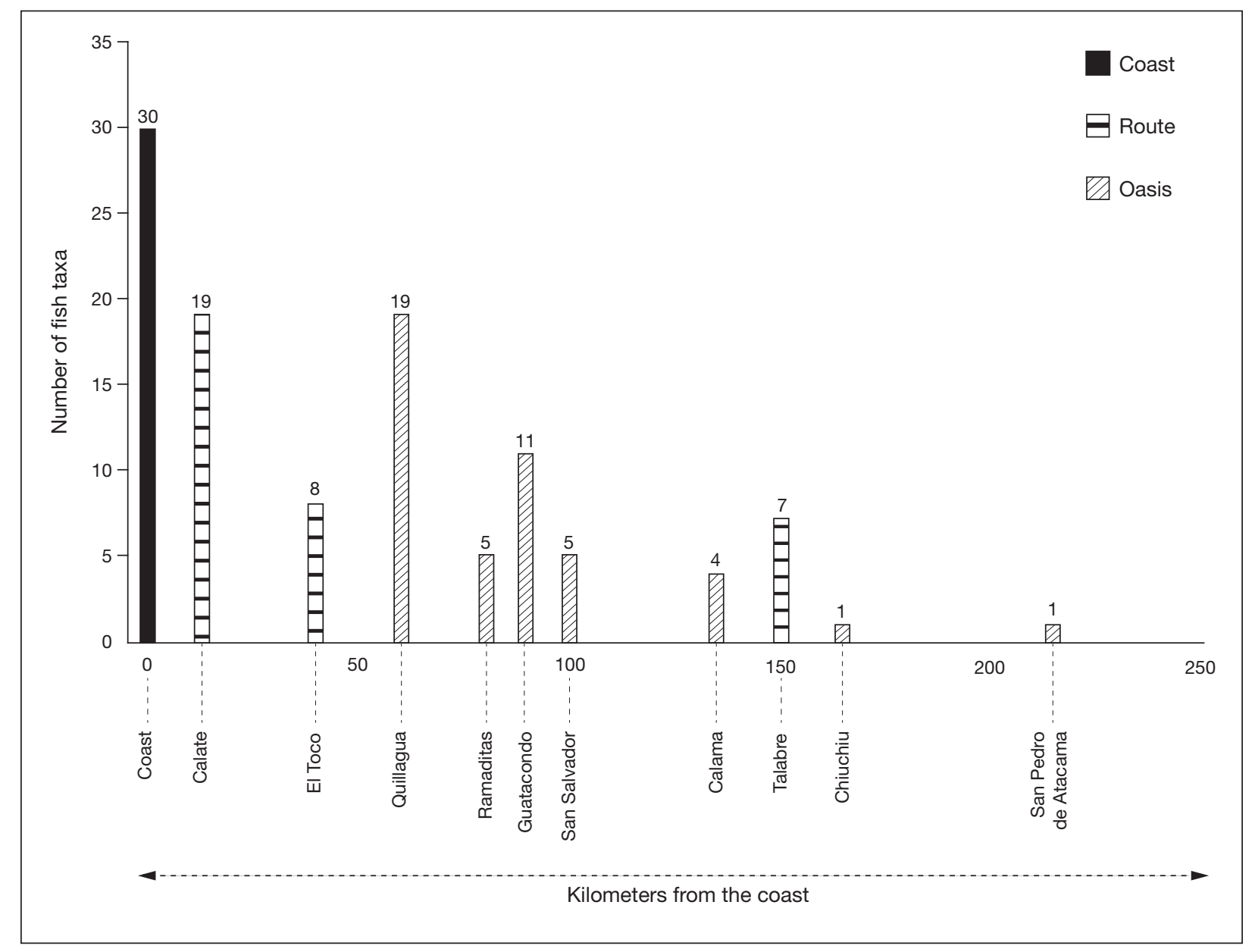

FIG. 8. - Graphic showing the taxonomic richness of the different localities from the Atacama Desert. It has been distinguished between coast, oasis and routes. For coastal sites column, we have employed the information available in publications about Formative period archaeological sites from Antofagasta region (Bravo 1981, 1985; Llagostera 1990; Castelleti 2007; Castelleti \& Maltraín 2010).

proportion of postcranial compared to cranial elements, which indicates that the fish were butchered or reduced prior to being transported to the interior oases. Head removal is understandable, given its low meat content and the decreased weight of the final transportable product, particularly for large fish ${ }^{4}$, where it appears to have been commonplace; it does not, however, appear to have been common for smaller species such as anchovy, which are found complete in interior sites (Gallardo 2017). From a taphonomic point of view, the low frequency of cranial and facial elements certainly cannot be ascribed to differential preservation, as these osteological elements are particularly dense (Falabella et al. 1994).

Regarding fish processing prior to transport, at least two distinct strategies can be identified: the transport of whole fish and that of butchered/headless fish. Both strategies were used in the Formative period to transport fish to the interior, with the choice of which depending on the species in question and distance to be traveled. While in Quillagua

4. Regrettably we do not have estimation size analyses for the all archaeological sites, but Castillo $(2011,2015)$ presents this information at least for the San Salvador village. and other coastal outposts in the desert such as Calate and El Toco, we confirmed the presence of complete specimens of different species (including cranial bones, but in low quantity), in places farther away from the coast such as Calama, Chiuchiu, San Pedro de Atacama, Guatacondo and San Salvador, all specimens except the anchovy appear butchered and headless (Fig. 9). The anchovy's special treatment is largely a result of its small size, as butchering and/or head removal would not only have resulted in the loss of much of the edible portion and therefore of much of its economic value, but would also have been an extremely labor-intensive task to process the large volume of fish captured by a system of nets. Even so, we cannot rule out the possibility that the differential treatment of the anchovy was not just a practical decision, but also a reflection of this species enormous (dual) social significance, as discussed above.

As in colonial and republican times (Feuillée 1714; Pernoud 1990; Ballester et al. 2010; Álvarez 2013), drying was no doubt the most common method of preserving fish after capture. Not only is drying a technique that would have required no additional investment (e.g. salt or wood), the Atacama Desert environment naturally favored the desiccation and 
TABLE 4. - Fish species presences in the coast, routes and interior archaeological sites. For coastal sites column we had employ the information available in publications about Formative period archaeological sites from Antofagasta region (Bravo 1981, 1985; Llagostera 1990; Castelleti 2007; Castelleti \& Maltraín 2010).

\begin{tabular}{|c|c|c|c|c|c|c|}
\hline Order/Family & Scientific name & Common name & Coast & Route & Quillagua & $\begin{array}{c}\text { Valleys, oases } \\
\text { and ravines }\end{array}$ \\
\hline \multicolumn{7}{|l|}{ Perciformes } \\
\hline Aplodactylidae & Aplodactylus punctatus Valenciennes, 1832 & Marblefish & $\times$ & $x$ & - & - \\
\hline Carangidae & Trachurus murphyi Nichols, 1920 & Jack mackerel & $x$ & $x$ & $x$ & $x$ \\
\hline Centrolophidae & Seriolella violacea Guichenot, 1848 & Palm ruff & $x$ & - & $x$ & $x$ \\
\hline Cheilodatylidae & Cheilodactylus variegatus Valenciennes, 1833 & Peruvian morwong & $x$ & $x$ & $x$ & $x$ \\
\hline \multirow[t]{2}{*}{ Haemulidae } & Anisotremus scapularis (Tschudi, 1846) & Peruvian grunt & $x$ & - & - & - \\
\hline & Isacia conceptionis (Cuvier, 1830) & Cabinza grunt & $x$ & $x$ & - & - \\
\hline Gempylidae & Thyrsites atun (Euphrasen, 1791) & Snoek & $x$ & - & - & - \\
\hline \multirow[t]{2}{*}{ Kyphosidae } & Girella laevifrons (Tschudi, 1846) & Sea chub & $x$ & - & - & - \\
\hline & Graus nigra Philippi, 1887 & Sea chub & $x$ & $x$ & $x$ & $x$ \\
\hline Labridae & Semicossyphus darwini (Jenyns, 1842) & Sheephead wrasse & $x$ & $x$ & $x$ & $x$ \\
\hline \multirow[t]{2}{*}{ Labrisomidae } & Auchenionchus sp. & Chalaco & - & $x$ & - & - \\
\hline & Labrisomus philippii (Steindachner, 1866) & Chalapo clinid & $x$ & $x$ & - & - \\
\hline Oplegnathidae & Oplegnathus insignis (Kner, 1867) & Pacific beakfish & $x$ & - & - & - \\
\hline \multirow[t]{2}{*}{ Pinguipedidae } & Pinguipes chilensis Valenciennes, 1833 & Chilean sandperch & $x$ & $x$ & $x$ & - \\
\hline & Prolatilus jugularis (Valenciennes, 1833) & Pacific sandperch & - & $x$ & - & - \\
\hline Pomacentridae & Chromis crusma (Valenciennes, 1833) & Damselfish & $x$ & - & - & - \\
\hline Scombridae & Sarda chiliensis (Cuvier, 1832) & Bonito & $x$ & $x$ & $x$ & $x$ \\
\hline \multirow[t]{3}{*}{ Serranidae } & Acanthistius pictus (Tschudi, 1846) & Brick seabass & $x$ & $x$ & $x$ & - \\
\hline & Paralabrax humeralis (Valenciennes, 1828) & Peruvian rock seabass & $x$ & - & $x$ & - \\
\hline & Hemilutjanus macrophthalmos (Tschudi, 1846) & Grape-eye seabass & $x$ & $x$ & $x$ & $x$ \\
\hline \multirow[t]{4}{*}{ Sciaenidae } & Cilus gilberti (Abbott, 1899) & Corvina drum & $x$ & $x$ & $x$ & $\times$ \\
\hline & Cynoscion analis (Jenyns, 1842) & Peruvian weakfish & $x$ & - & - & - \\
\hline & Sciaena deliciosa (Tschudi, 1846) & Lorna drum & $x$ & - & $x$ & - \\
\hline & Sciaena sp. & Sargo jote & $x$ & - & - & - \\
\hline Xiphiidae & Xiphias gladius Linnaeus, 1758 & Swordfish & $x$ & - & $x$ & - \\
\hline \multicolumn{7}{|l|}{ Chimaeriformes } \\
\hline Callorhinchidae & Callorhinchus callorynchus (Linnaeus, 1758) & Plownose chimaeras & - & $x$ & - & - \\
\hline \multicolumn{7}{|l|}{ Clupeiformes } \\
\hline Engraulidae & Engraulis ringens Jenyns, 1842 & Anchovy & $x$ & $x$ & $x$ & $x$ \\
\hline Clupeidae & Ethmidium maculatum (Valenciennes, 1847) & Pacific Menhaden & $x$ & $x$ & - & - \\
\hline \multicolumn{7}{|l|}{ Ophidiiformes } \\
\hline Ophidiidae & Genypterus sp. & Cusk-eel & $x$ & - & $x$ & $x$ \\
\hline \multicolumn{7}{|l|}{ Gobiesociformes } \\
\hline \multicolumn{7}{|c|}{ Pleuronectiformes } \\
\hline Paralichtyidae & Paralichthys microps (Günther, 1881) & Flounder & $x$ & $x$ & $x$ & $x$ \\
\hline \multicolumn{7}{|l|}{ Scorpaeniformes } \\
\hline Sebastidae & Sebastes oculatus Valenciennes, 1833 & Patagonian redfish & $x$ & $x$ & $x$ & $x$ \\
\hline \multicolumn{7}{|l|}{ Lamniformes } \\
\hline Lamnidae & Isurus oxyrinchus Rafinesque, 1810 & Shortfin mako shark & - & - & $x$ & - \\
\hline \multicolumn{7}{|l|}{ Indeterminate } \\
\hline \multirow[t]{2}{*}{ Indeterminate } & Squalimorpha indet. & Shark indet. & - & - & $x$ & - \\
\hline & Chondrichthyes indet. & - & $x$ & - & - & - \\
\hline Total & & & 30 & 19 & 19 & 12 \\
\hline
\end{tabular}

dehydration of organic matter, making the drying of fish a simple task (Castillo 2011, 2015). This food preparation process required a greater labor investment to preserve and transport the product, and as such it represented an economic goal that went beyond immediate consumption to include long term planning for deferred consumption (Woodburn 1982), whether by the fishermens' communities themselves or by other groups that ultimately obtained and consumed those fish. Coastal groups intentionally became part of extra-local exchange networks, assuming a leading role in the production (exploitation-processing) and distribution of dried fish to become active agents in the economy of the Atacama Desert region during the Formative period.

\section{CONCLUSIONS}

The Formative period was an era of profound social transformation throughout the Atacama Desert, and those changes manifested themselves differently according to distinct regional and local histories, processes, and experiences. Perhaps the most definitive feature of this period - in social terms - is the expansion of spheres of interaction and the strengthening of ties among geographically distant communities (Agüero et al. 2006; Núñez et al. 2006; Uribe 2009; Castro et al. 2016; Gallardo et al. 2017a). This new framework of relations transformed local and traditional identities by exposing them to a new cosmopolitan reality through a constantly 


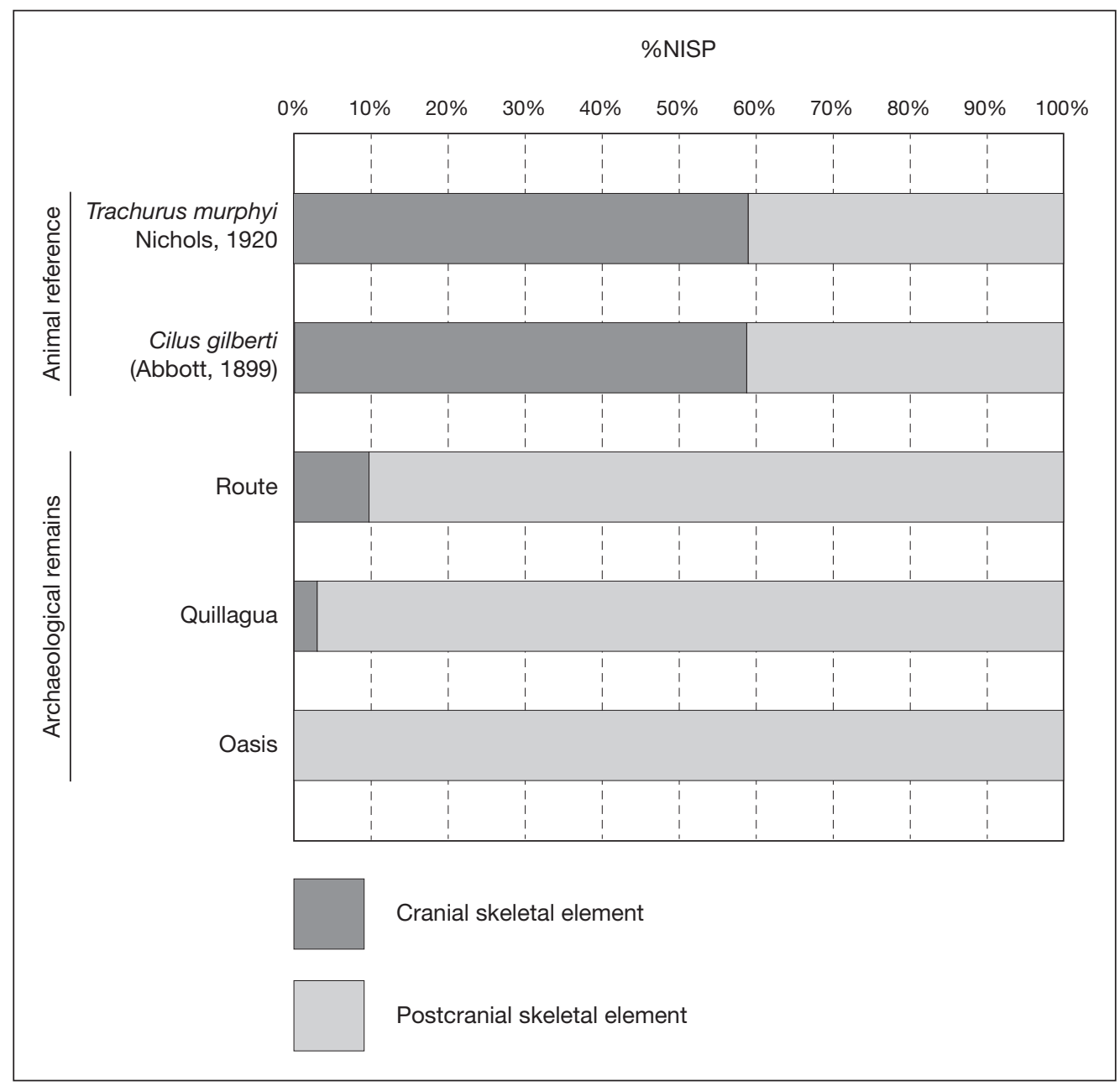

FIG. 9. - Graphic that indicates the relation between cranial and postcranial skeletal units in routes, Quillagua and interior oasis sites from the Atacama Desert during the Formative period. Both inferior bars show the same relation in reference skeletons from Trachurus murphyi Nichols, 1920 (60 cranial and 41 postcranial elements) and Cilus gilberti (Abbott, 1899) (60 cranial and 42 postcranial elements). Abbreviation: NISP, number of identified specimens.

changing panorama of traveling outsiders, exotic goods, new habitus, and novel customs that revealed and enabled new ways of being and doing. That atmosphere allowed groups, sectors, and communities to reinvent their cultures and may have catalyzed the emergence of individual and collective differentiation - a sociocultural tool for establishing political agency on a local and/or regional scale.

It is in precisely this sociopolitical and economic context that fish enters the game as a central player. As a proprietary product arising purely from coastal agency, during the Formative period this resource became increasingly attractive outside the geographical and social boundaries of the coast (Castillo 2011, 2015; Ballester \& Clarot 2014; Gallardo et al. 2017a). It served as food along trade routes and in the oases for both coastal groups and agropastoralists of the interior. On the coast, consumption was intensive, as fish was a dietary staple that was consumed raw, dried, or cooked, butchered or whole (Arriaza 1995; Reinhard \& Urban 2003; Ardiles et al. 2011; Pestle et al. 2015a). Owing to the distances involved, the sometimes precarious means of transport, storage limitations, and the desert conditions, in the interior fish was consumed dried, on a small scale, and was not necessarily available in equal quantity to all community members; rather, it was generally a compliment to food produced locally by gathering, horticulture, farming, and hunting (Rivera 2002; Pestle et al. 2015a, b; Pestle 2017).

In this regard we propose that fish, as a foreign and exotic product, considered as material culture, played a major role in the intralocal relations by supporting the construction and reproduction of internal differences among the collectives living in the inland desert valleys and oases, distinguishing between those who had and did not have access to this product and to what degree (Van der Veen 2003). This value was also expressed outside of the strictly dietary realm, as fish was a constant feature in funerary offerings, which demonstrates its polysemic value and its active multifaceted social role. Thus, beyond the economic plane, the adoption of a new culinary element transformed food preferences (Smith 2006) among interior agropastoralists, thereby modifying their own identity in response to new intercultural relations (Dietler 2007), as they forged closer ties with coastal groups without losing the elements of their own tradition. This phenomenon represents 
the formation of a new cultural entity at the culinary level, constructed from local and foreign elements (Barthes 1961). A similar social and cultural phenomenon occurs in the other sense, as during the Formative period littoral collectives incorporate foreign cultivated vegetables and ceramic vessel produced by inland populations, completely changing part of their coastal culinary traditions as a way to create cultural affinities for maintain and reproduce their social ties with their interior neighbors (Carrasco et al. 2017; Correa et al. 2018).

On the macroregional scale, this phenomenon creates a culinary distinction between those who lived close to the Pacific and those residing in the interior, but more importantly, it testifies to their strong economic linkage and the cultural negotiations they had to engage in to maintain that bond. Thus, a new social structure and relational network based on food circulation emerged, in association with other kinds of goods, products, persons, and ideas. The economic cycle of fish, specifically, began with those who lived on the coast and was maintained and expanded over time as a consequence of the high culinary value assigned to fish by the people living in the Atacama Desert interior. In this context, dried fish did not merely satisfied a nutritional need; it became yet another element of the material culture that groups employed to maintain, strengthen, and reproduce the relations they had established with far away communities; furthermore, it provided a new avenue for marking distinctions between certain individuals and groups within the very heart of those inland communities.

\section{Acknowledgements}

FONDECYT 1160045 and 1090762, FONDAP 15110006.

This article is an extended and more complete version of a previous work of the research team published in a monographic series (Castillo et al. 2017). We especially thank Mariana Ugarte and Charles Rees. We also express our gratitude to the two anonymous referees whose insightful comments and suggestions have helped us to improve this article.

\section{REFERENCES}

Adán L., Urbina S., Pellegrino C. \& Agüero C. 2013. - Aldeas en los bosques de Proso+--pis. Arquitectura residencial y congregacional en el período Formativo tarapaqueño (900 AC-900 DC). Estudios Atacameños (45): 75-94. https://doi.org/10.4067/ S0718-10432013000100006

AgÜERO C. \& CASES B. 2004. - Quillagua y los textiles Formativos del Norte Grande de Chile. Chungara, Revista de Antropología Chilena Volumen Especial: 599-617.

Agǘro C. \& Uribe M. 2011. - Las sociedades Formativas de San Pedro de Atacama: Asentamiento, cronología y proceso. Estudios Atacameños (42): 53-78. https://doi.org/10.4067/S071810432011000200004

Agüero C., Uribe M., Ayala P., Cases B. \& Carrasco C. 2001. Ceremonialismo del periodo Formativo en Quillagua, Norte Grande de Chile. Boletin de la Sociedad Chilena de Arqueología 32: $24-34$

Agüero C., Ayala P., Uribe M., Carrasco C. \& Cases B. 2006. El periodo formativo desde Quillagua, Loa inferior (norte de
Chile), in LECHMAN H. (ed.), Esferas de interacción prehistóricas $y$ fronteras nacionales modernas: los Andes sur centrales. Instituto de Estudios Peruanos, Lima: 73-118.

Álvarez O. 2013. - El último constructor de balsas de Cuero de Lobo. Ediciones Mediodía en Punto, Vallenar, 45 p.

Araújo A., Reinhard K., Leles D., Sianto L., IÑiguez A., Fugassa M., Arriza B., Orellana N. \& Ferreira L. 2011. - Paleoepidemiology of intestinal parasites and lice in pre-columbian South America. Chungara, Revista de Antropología Chilena 43 (2):303-313.

Ardiles H., Ballester B. \& Clarot A. 2011. — Elección de dieta en poblaciones pasadas costeras de la II región: una mirada multidisciplinaria. Informes FAIP 14: 83-110.

ARRIAZA B. 1995. - Chinchorro bioarchaeology: chronology and mummy seriation. Latin American Antiquity 6 (1): 35-55.

Ayala P. 2001. - Las sociedades formativas del Altiplano Circumtiticaca y Meridional y su relación con el Norte Grande de Chile. Estudios Atacameños 21: 7-39.

BALlester B. 2017. - La pesca y la caza marina en el desierto de Atacama: luces conceptuales desde los documentos escritos (siglos XVI-XIX). CUHSO, Cultura-Hombre-Sociedad 27 (2): 89-120. https://doi.org/10.7770/cuhso-v27n2-art1206

BALLESTER B. 2018. - Tecnología de arponaje en la costa del desierto de Atacama, norte de Chile. Estudios Atacameños 57: 65-95. https://doi.org/10.4067/S0718-10432018005000401

Ballester B. \& Clarot A. 2014. - La Gente de los Túmulos de Tierra. Marmot Impresores, Santiago, 140 p.

Ballester B. \& Crisóstomo M. 2017. - Percutores líticos de la pampa del desierto de Atacama (norte de Chile): Tecnología, huellas de uso, decoración y talladores. Chungara, Revista de Antropologia Chilena 49 (2): 175-192. https://doi.org/10.4067/ S0717-73562017005000016

Ballester B. \& Gallardo F. 2011. - Prehistoric and historic networks on the Atacama Desert coast (northern Chile). Antiquity 85: 875-889. https://doi.org/10.1017/S0003598X0006837X

Ballester B., San Francisco A. \& Gallardo F. 2010. - Modo de vida y economía doméstica de las comunidades cazadoras recolectoras costeras del Desierto de Atacama en tiempos coloniales y republicanos. Taltalia 3: 21-32.

BARTHES R. 1961. - Pour une psycho-sociologie de l'alimentation contemporaine. Annales. Économies, Sociétés, Civilisations 16 (5): 977-986. https://doi.org/10.3406/ahess.1961.420772

BENAVENTE A. 1978. - Chiu-chiu 200: poblado agroalfarero temprano. Revista Chilena de Antropología 1: 5-15.

Benavente A. 1981. - Chiu-chiu 200: un Campamento de Pastores. Degree dissertation, Universidad de Chile, Santiago [unpublished].

BenAVENTE A. 1988-1989. - Nuevas evidencias arqueológicas acerca de los asentamientos tempranos en el Loa Medio. Paleoetnológica 5: 65-70.

Benavente A. 1998. - Excavaciones de los sitios Chiuchiu 273-274. Informe Final Fondecyt 1950346. [unpublished].

BERENGUER J. 2008. - La costa: un lugar para vivir, in BERENGUER J. (ed.), Pescadores en la Niebla, los changos y sus ancestros. Museo Chileno de Arte Precolombino, Santiago: 23-29.

Berenguer J. \& Pimentel G. 2010. — Arqueología de los "espacios vacíos": una aproximación intermodal a las relaciones intersocietales, in Actas del XVII Congreso Nacional de Arqueología Chilena, Tomo II. Kultrún, Valdivia: 1305-1308.

BINFORD L. 1978. - Nunamiut Ethnoarchaeology. Academic Press, New York, 509 p.

BIRD J. B. 1943. - Excavations in northern Chile. Anthropological Papers of the American Museum of Natural History 38 (4): 173-318.

BIRD J. B. 1946. - The cultural sequence of the north Chilean coast, in STEWARD J. (ed.), Handbook of South American Indians. Vol. II. Smithsonian Institution, Washington: 587-594.

BitTMANN B. 1978. - Fishermen, mummies and balsa rafts on the coast of northern Chile. El Dorado 3 (3): 60-103.

BitTmann B. 1984. - El Proyecto Cobija: investigaciones antropológicas en la costa del Desierto de Atacama, in Actas 
Simposio de Arqueología Atacameña XLIV Congreso Internacional de Americanistas. Universidad del Norte, Antofagasta: 99-146.

Blanco J., MaZA M. \& ReES C. 2010. — Cazadores recolectores costeros y el aprovisionamiento de recursos líticos. Perspectivas interpretativas de los eventos de talla en el desierto absoluto. Werkén 13 (2): 45-68.

Blanco J., Correa I., Flores C. \& Pimentel G. 2017. — La extracción prehispánica de recursos minerales en el internodo Quillagua-Costa, desierto de Atacama. Estudios Atacameños (56): 77-102. https://doi.org/10.4067/S0718-10432017005000003

Boisset G., Llagostera A. \& Salas E. 1969. - Excavaciones arqueológicas en Caleta Abtao. Antofagasta, in Actas del V Congreso Nacional de Arqueología. DIBAM, La Serena: 75-152.

BraVo L. 1981. - Abtao-5: un modelo de adaptación tardía a la costa de la Segunda Región. Degree dissertation, Universidad del Norte, Antofagasta, 360 p.

BRAVO L. 1985. - Una proyección representative de los recursos ictiotróficos del yacimiento Abtao-5 y sus implicancias socioeconómicas, in Actas del IX Congreso Nacional de Arqueología. SCHA \& Museo Arqueológico de La Serena, La Serena: 95-104.

Burgess G., CuTTING C., LOVERN J. \& WATERMAN J. 1971. — El pescado y las industrias derivadas de la pesca. Acribia, Zaragoza, 392 p.

CALlen E. \& CAMERON T. 1960. - A prehistoric diet revealed in coprolites. The New Scientist 8 (190): 35-40.

CAPDEVIlle A. 1928. - Como descubrí la industria paleolítica americana de los sílices negros tallado en la zona de la costa de Taltal. Revista Chilena de Historia Natural 32: 348-364.

Carrasco C., Echeverría J., Ballester B. \& Niemeyer H. 2015. - De pipas y sustancias: costumbre fumatorias durante el período Formativo en el litoral del Desierto de Atacama (Norte de Chile). Latin American Antiquity 26 (2): 143-161. https:// doi.org/10.7183/1045-6635.26.2.143

Carrasco C., Correa I., Belmar C., Ballester B. \& Gallardo F. 2017. - Cocinando relaciones interculturales: residuos adheridos en vasijas cerámicas de grupos cazadores recolectores marinos del desierto de Atacama (Período Formativo, norte de Chile). Estudios Atacameños (55): 85-108. https://doi.org/10.4067/ S0718-10432017005000009

Cartajena I. 1994. - Determinación de restos óseos de camélidos en dos yacimientos del Loa Medio (II Región). Estudios Atacameños 11: 25-52.

Cartajena I., Núñez L. \& Grosjean M. 2007. — Camelid domestication on the western slope of the Puna de Atacama, northern Chile. Anthropozoologica 42 (2): 155-174.

Carvalho M., Araújo A. \& Ferrerira D. 2003. - Human intestinal parasites in the past: new findings and a review. Memórias do Instituto Oswaldo Cruz 98 (Suppl. 1): 103-118.

Cases B., Rees C., Pimentel G., Labarca R. \& Leiva D. 2008. Sugerencias desde un contexto funerario en un "espacio vacío" del desierto de Atacama. Boletín del Museo Chileno de Arte Precolombino 13 (1): 51-70. https://doi.org/10.4067/S071868942008000100004

CASTEEL R. 1980. - A preliminary investigation of fish remains in midden material from Northern Chile, in MEIGHAN C. \& TRUE D. (eds), Prehistoric trails of Atacama: Archaeology of Northern Chile. Los Angeles Institute of Archaeology, University of California, Los Angeles: 179-187.

Castelleti J. 2007. - Patrón de asentamiento y uso de recursos a través de la secuencia ocupacional prehispánica en la costa de Taltal. Master dissertation, Universidad Católica del Norte, Antofagasta, 100 p.

Castelleti J. \& Maltrain G. 2010. — El formativo de Taltal y el patrón de asentamiento local, in Actas del XVII Congreso Nacional de Arqueología Chilena, Tomo I. Kultrún, Valdivia: 165-176.

Castells C., González C. \& Westrall C. 2010. — Endoparasitismo y dieta en dos poblaciones atacameñas: análisis de coprolitos de los sitios Mina Las Turquesas y Cementerio Regimiento Chorrillos, in Actas del XVII Congreso Nacional de Arqueología Chilena, Tomo II. Kultrún, Valdivia: 759-768.
Castillo C. 2011. - La aldea San Salvador y la circulación del pescado en el Formativo Medio (500 AC-100 DC) en la Región de Antofagasta. Degree dissertation, Universidad Internacional SEK, Santiago, $118 \mathrm{p}$

CASTILlo C. 2015. - El ciclo económico del pescado en el Formativo medio en la región de Antofagasta, norte de Chile: análisis ictiológico del sitio Aldea San Salvador, in Actas del XIX Congreso Nacional de Arqueologia Chilena. UTA-SCHA, Arica: 401-404.

Castillo C., Ballester B., Calás E., Labarca R. \& Gallardo F. 2017. — La ruta de los peces más allá del litoral: sobre el ciclo del pescado seco en el desierto de Atacama (periodo Formativo), in Gallardo F., Ballester B. \& FuenZalida N. (eds.), Monumentos funerarios de la costa del desierto de Atacama. Los cazadores-recolectores marinos y sus intercambios (500 A.C.-700 D.C.). CIIR \& SCHA, Santiago: 55-65.

Castro V., Berenguer J., Gallardo F., Llagostera A. \& SalaZAR D. 2016. - Vertiente occidental circumpuneña. Desde las sociedades posarcaicas hasta las preincas ( $c a .1 .500$ años A.C. a 1.470 ańos D.C.), in Falabella F., Uribe M., Sanhueza L., Aldunate C. \& Hidalgo J. (eds), Prehistoria en Chile. Desde sus primeros habitantes hasta los Incas. Editorial Universitaria, Santiago: 239-283.

Clarke J. 2006. - Antiquity of aridity in the Chilean Atacama Desert. Geomorphology 73: 101-114.

Contreras R., Núñez P., Llagostera A., Cruz J., San Francisco A., Ballester B., Rodríguez O. \& Becerra G. 2011. - Un conglomerado del período Arcaico costero Medio del área Taltal Paposo, Norte de Chile. Taltalia (4): 7-31.

Correa I., Carrasco C., Ballester B. \& Gallardo F. 2018. Efectos colaterales de la transición al Formativo: una nueva culinaria entre los cazadores-recolectores marinos del desierto de Atacama. Chungara, Revista de Antropología Chilena 50 (1): 87-106. https://doi.org/10.4067/S0717-73562018005000101

DietLer M. 2007. - Culinary encounters: food, identity, and colonialism, in TwISs K. (ed.), The Archaeology of Food and Identity. Press University of Southern Illinois, Carbondale: 218-242.

DrUSS M. 1978. - Environment, subsistence economy and settlement pattern of the Chiu Chiu complex (c. 2700-1600 BC) of the Atacama Desert, northern Chile. PhD dissertation, Columbia University, 287 p.

Falabella F., Vargas L. \& Meléndez R. 1994. — Differential preservation and recovery of fish remains in Central Chile. Annales $d u$ Musée royal de l'Afrique centrale, Sciences Zoologiques 274: 25-35.

Falabella F., MELÉNDEZ R. \& VARGAS L. 1995. - Claves osteológicas para peces de Chile central. Un enfoque arqueológico. Artegrama Ltda., Chile, 208 p.

Ferreira L., Araújo A., Confaloneri U. \& NúN̄ez L. 1984. The finding of eggs of Diphyllobothrium in human coprolites (4,100-1950 B.C.) from northern Chile. Memórias do Instituto Oswaldo Cruz 79 (2): 175-180.

FEUILLÉE L. 1714. - Journal des observations physiques, mathematiques et botaniques, faites par l'ordre du Roy fur les Côtes Orientales de l'Amerique Meridionale, et dans les Indes Occidentales, depuis l'année 1707 jufques en 1712. Pierre Giffart, Paris, 767 p.

Flores C., Figueroa V. \& Salazar D. 2016. — Middle Holocene production of mussel shell fishing artifacts on the coast of Taltal ( $25^{\circ}$ Lat South), Atacama desert, Chile. The Journal of Island and Coastal Archaeology 11 (3): 411-424. https://doi.org/10.1080/1 5564894.2015 .1105884

Flores H. \& RENDIC J. 2011. - Conducta alimenticia, supervivencia y crecimiento de juveniles silvestres de Graus nigra Philippi, 1887 en cautiverio (Perciformes: Kyphosidae). Latin American Journal of Aquatic Research 39 (3): 607-612. https:// doi.org/10.3856/vol39-issue3-fulltext-21

FOLLET W. 1980. - Fish remains from the archaeological site of Guatacondo, Chile, in Meighan C. \& True D. (eds), Prehistoric Trails of Atacama: Archaeology of Northern Chile. Los Angeles Institute of Archaeology, University of California, Los Angeles: 135-138. 
Fuentes H. 1981a. - Feeding habit of Semicossyphus maculatus (Labridae) in coastal waters of Iquique in northern Chile. Japanese Journal of Ichthyology 27 (4): 309-315.

Fuentes H. 1981b. - Nicho alimentario de Pimelometopon maculatus (Pérez, 1886) (piscis; Labridae) en Playa Blanca, Iquique. Boletín de la Sociedad de Biología de Concepción 51: 109-117.

FuENTES H. 1982. - Feeding habits of Graus Nigra (Labridae) in coastal waters of lquique in northern Chile. Japanese Journal of Ichthyology 29 (1): 95-98.

GALLARDO F. 1993. - La sustancia privilegiada: turbantes, poder y simbolismo en el Formativo del norte de Chile, in BeRENGUER J. (ed.), Identidad y prestigio en Los Andes. Museo Chileno de Arte Precolombino, Santiago: 9-15.

GALLARDO F. 2017. - Arqueología de los intercambios recíprocos: Costa y oasis del río loa medio e inferior, época formativa (500 cal A.C.-700 cal D.C.), in GALlardo F., Ballester B. \& FueNZALIDA N. (eds), Monumentos funerarios de la costa del desierto de Atacama. Los cazadores-recolectores marinos y sus intercambios (500 A.C.-700 D.C.). ICIIS \& SCHA, Santiago: 15-24.

Gallardo F. \& Cabello G. 2015. — Emblems, leadership, social interaction and early social complexity: the ancient Formative period (1500 BC-AD 100) in the desert of northern Chile. Cambridge Archaeological Journal 25 (3): 615-634. https://doi. org/10.1017/S0959774315000013

GALLARDO F. \& SOUZA P. DE 2008. — Rock art, modes of production, and social identities during the Early Formative period in the Atacama desert (northern Chile), in SANZ I., FIORE D. \& MaY S. (eds), Archaeologies of Art. Time, Place, Identity. Routledge, London, New York: 79-97. (Coll. One world archaeology; 55).

Gallardo F. \& Yacobaccio H. 2005. - Wild or domesticated? Camelids in Early Formative rock art of the Atacama desert (northern Chile). Latin American Antiquity 16 (2): 115-130. https://doi.org/10.2307/30042807

Gallardo F., Cornejo L., SÁnchez R., Cases B., Román A. \& DezA A. 1993a. - Arqueología en el valle de Quillagua, río Loa, Norte de Chile. Gaceta Arqueológica Andina 23: 125-138.

Gallardo F., Cornejo L., Sánchez R., Cases B., Román A. \& DEZA A. 1993b. - Una aproximación a la cronología y el asentamiento en el oasis de Quillagua (río Loa, II Región), in Actas del XII Congreso Nacional de Arqueología Chilena, Tomo 2. Museo Regional de la Araucanía; Sociedad Chilena de Arqueología, Temuco: 41-60.

Gallardo F., Ballester B. \& Fuenzalida N. 2017a. - Monumentos funerarios y flujos de información social costera, in GALLARDO F., BALlester B. \& FuENZALIDA N. (eds), Monumentos funerarios de la costa del desierto de Atacama. Los cazadores-recolectores marinos y sus intercambios (500 A.C.-700 D. C.). ICIIS \& SCHA, Santiago: 39-54.

Gallardo F., Correa I., Blanco J. \& Pimentel G. 2017b. Consumption consumes: circulation, exchange, and value of San Pedro de Atacama black polished ceramics. Latin American Antiquity 28 (2): 252-268. https://doi.org/10.1017/laq.2017.15

GonZÁlez C. \& WestFall C. 2010. - Cementerio Regimiento Chorrillos de Calama: testimonios funerarios formativos en el Loa Medio, región de Antofagasta, in Actas del XVII Congreso Nacional de Arqueología Chilena, Tomo I. Kultrún, Valdivia: 95-105.

GraYson D. 1984. — Quantitative Zooarchaeology. Academic Press, Orlando, $202 \mathrm{p}$.

HASTORF C. 2012. - The habitus of cooking practices at Neolithic Çatalhöyük: what was the place of the cook?, in GRAFF S. \& Rodríguez E. (eds), The Menial Art of Cooking. Archaeological Studies of Cooking and Food Preparation. University Press of Colorado, Colorado: 65-86.

Hoffman B., CZederplitz J. \& PARTlow M. 2000. — Heads or tails: the zooarchaeology of Aleut salmon storage on Unimak island, Alaska. Journal of archaeological science 27 (8): 699-708. https://doi.org/10.1006/jasc.1999.0492

Hogg A., Hua Q., Blackwell P., Niu M., Buck C, Guilderson
T., Heaton T., Palmer J., Reimer P., Reimer R., Turney C. \& Zimmerman S. 2013. - SHCal13 Southern Hemisphere Calibration, 0-50,000 Years cal BP. Radiocarbon 55 (4): 1889-1903. https://doi.org/10.2458/azu_js_rc.55.16783

Jiménez F. 1943. - La industria de la pesca. Principios de oceanografía, técnicas de la pesca, industria de la pesca en tierra, industrias afines y derivadas. Nacimiento, Santiago, $314 \mathrm{p}$.

Knudson K., Pestle W., Torres-Rouff C. \& Pimentel G. 2012. - Assessing the life history of an andean traveller through biogeochemistry: stable and radiogenic isotope analyses of archaeological human remains from Northern Chile. International Journal of Osteoarchaeology 22 (4): 435-451. https://doi. org/10.1002/oa. 1217

Labarca R., Calás E., Gallardo F., Ballester B. \& Prieto A. 2015. - Chaetophractus vellerosus Gray, 1865 (Xenarthra, Dasypodidae) en un cementerio de túmulos de la desembocadura del río Loa (Región de Antofagasta, Chile): evidencias de conexiones con el altiplano andino, periodo Formativo Tardío (500 A.C.800 D.C.). Estudios Atacameños (50): 47-58.

Labarca R., Calás E. \& Prieto A. 2017. — Los artefactos óseos de contextos funerarios costero de la región de Antofagasta, in Gallardo F., Ballester B. \& FuenZAlida N. (eds), Monumentos funerarios de la costa del desierto de Atacama. Los cazadores-recolectores marinos y sus intercambios (500 A.C.-700 D.C.). ICIIS \& SCHA, Santiago: 95-106.

LATCHAM R. 1909. - El comercio precolombino en Chile y otros países de América. Anales de la Universidad de Chile 125: 241-284.

LATCHAM R. 1910. - Los changos de las costas de Chile. Imprenta Cervantes, Santiago, $65 \mathrm{p}$.

Latcham R. 1938. - Arqueología de la Región Atacameña. Prensas de la Universidad de Chile, Santiago, 374 p.

LATORRE E. 2017. - Los metales de los túmulos formativos: una primera aproximación, in GALLARDO F., BALLESTER B. \& FUENZALIDA N. (eds), Monumentos funerarios de la costa del desierto de Atacama. Los cazadores-recolectores marinos y sus intercambios (500 A.C.-700 D.C.). ICIIS \& SCHA, Santiago: 169-182.

Le Paige G. 1977. - Recientes descubrimientos arqueológicos en la zona de San Pedro de Atacama. Estudios Atacameños 5: 111-126.

Llagostera A. 1989. - Caza y pesca marítima, in Hidalgo J., Schiappacasse V., Niemeyer H., Aldunate C. \& Solimano I (eds), Prehistoria. Desde sus origenes hasta los albores de la conquista. Andrés Bello, Santiago: 57-81.

Llagostera A. 1990. - La navegación prehispánica en el Norte de Chile: bioindicadores e inferencias teóricas. Chungara, Revista de Antropología Chilena (24-25): 37-51.

Llagostera A. 2013. - Poblaciones marítimas con arquitectura. Hombre y Desierto 17: 151-183.

Marquet P., Bozinovic F., Bradshaw G., Cornelius C., GonzÁlez H., Gutierres J., Hajek E., Lagos J., López-Cortez J., NúNÉEZ L., Rosello E., Santoro C., Samaniego H., Standen V., TORRES-Mura J. \& JAKSIC F. 1998. — Los ecosistemas del desierto de Atacama y área andina adyacente en el norte de Chile. Revista Chilena de Historia Natural 71: 593-617.

McKay C., Friedmann I., GÓmeZ-Silva B., CÁCERES-Villanueva L., Andersen D. \& LANDheim R. 2003. - Temperature and moisture conditions for life in the extreme arid region of the Atacama Desert: four years of observations including the El Niño of 1997-1998. Astrobiology 3 (2): 393-406. https://doi. org/10.1089/153110703769016460

MatTe J. 1981. — Misión en el Paposo. Teología y Vida 22 (1): 51-64. Medina M. \& Arancibia H. 2002. — Dinámica trófica del jurel (Trachurus symmetricus murphyi) en el norte de Chile. Investigaciones Marinas 30 (1): 45-55. https://doi.org/10.4067/S071771782002000100003

Meighan C. \& True D. 1980. - Prehistoric trails of Atacama: archaeology of Northern Chile. The Institute of Archaeology of the University of California, Los Angeles, 228 p. (Coll. Monumenta Archaeologica; 7). 
Moragas C. 1982. - Túmulos funerarios de la costa sur de Tocopilla (Cobija), II Región. Chungara, Revista de Antropología Chilena 9: 152-173

Mostny G. 1942. - Informe preliminar sobre las excavaciones efectuadas en la costa chilena entre Pisagua y Coquimbo del 8 de Octubre de 1941 al 15 de Marzo de 1942. Boletín del Museo Nacional de Historia Natural 20: 97-102.

MostNy G. 1964a. - Anzuelos de concha: 6170 220 años. Noticiario Mensual del Museo Nacional de Historia Natural 95: 7-8.

Mostny G. 1964b. - Arqueología de Taltal: epistolario de Augusto Capdeville con Max Uhle y otros. Fondo Histórico y Bibliográfico José Toribio Medina, Santiago, 360 p.

Mostny G. 1970a. - La subárea arqueológica de Guatacondo. Boletín del Museo Nacional de Historia Natural 29: 271-287.

Mostny G. 1970b. - Arqueología de la Quebrada de Guatacondo. Órbita Revista de Ciencia y Tecnología 6: 6-20.

Mostny G. \& Niemeyer H. 1963. — Informe sobre investigaciones arqueológicas en la quebrada de Guatacondo. Noticiario Mensual Museo Nacional de Historia Natural 86: 2-6.

NúNEZZ L. 1971. - Secuencia y cambio en los asentamientos humanos de la desembocadura del Río Loa, en el Norte de Chile. Boletín de la Universidad de Chile 112: 2-25.

NúNEZZ L. 1985. - Tráfico de complementariedad de recursos entre las tierras altas y el Pacifico en el área Centro-Sur Andina. PhD dissertation, University of Tokyo [unpublished].

NúNez L. \& Dillehay T. 1979. - Movilidad Giratoria, Armonía Social y Desarrollo en los Andes Meridionales: Patrones de Tráfico e Interacción Económica. Ensayo. Universidad Católica del Norte, Antofagasta, $170 \mathrm{p}$

NúNEZZ L., Zlatar V. \& NúÑEZ P. 1974. — Caleta Huelén 42: una aldea temprana en el norte de Chile (nota preliminar). Hombre y Cultura 2 (5): 67-103.

Núñez L., Cartajena I., Carrasco C. \& Souza P. de 2005. - El templete de Tulán y sus relaciones formativas panandinas (Norte de Chile). Boletín del Instituto Francés de Estudios Andinos 34: 299-320.

Núñez L., Cartajena I., Carrasco C., Souza P. de \& Grosjean M. 2006. - Emergencia de comunidades pastoralistas formativas en el sureste de la Puna de Atacama. Estudios Atacameños 32: $93-117$

Orellana M. 1991. - Contextos culturales tempranos de Toconao Oriente. Diálogo Andino 10: 22-46.

Ortlieb L. 1995. - Paleoclimas cuaternarios en el norte grande de Chile, in Argollo J. \& Mourguiart P. (eds), Cambios cuaternarios en América del Sur. ORSTOM, La Paz: 225-246.

Pernoud R. 1990. - América del Sur en el siglo XVIII. Misceláneas anecdóticas y bibliográficas. Fondo de Cultura Económica, México, 163 p.

Pestle W. 2017. - Living, eating, and dying in the Formative period Atacama, in Gallardo F., Ballester B. \& FuenZALida N. (eds), Monumentos funerarios de la costa del desierto de Atacama. Los cazadores-recolectores marinos y sus intercambios (500 A.C.-700 D.C.). ICIIS \& SCHA, Santiago: 209-224.

Pestle W., Torres-Rouff C., Gallardo F., Ballester B. \& Clarot A. 2015a. - Mobility and exchange among marine hunter-gatherer and agropastoralist communities in the Formative Period Atacama Desert. Current Anthropology 15 (1): 121-133.

Pestle W., Torres-Rouff C., Hubbe M., Santana F., Pimentel G., Gallardo F. \& KnUdSOn K. 2015b. - Explorando la diversidad dietética en la prehistoria del desierto de Atacama: un acercamiento a los patrones regionales. Chungara, Revista de Antropologia Chilena 47 (2): 201-209. https://doi.org/10.4067/ S0717-73562015005000013

Pimentel G. 2012. - Redes viales prehispánicas en el desierto de Atacama. Viajeros, Movilidad e Intercambio. PhD dissertation, Universidad Católica del Norte y Universidad de Tarapacá, 398 p.

Pimentel G. \& UGarte M. 2017. — La agencia costera en la Pampa del desierto de Atacama, in Gallardo F., Ballester
B. \& FuenZALida N. (eds), Monumentos funerarios de la costa del desierto de Atacama. Los cazadores-recolectores marinos y sus intercambios (500 A.C.-700 D.C.). ICIIS \& SCHA, Santiago: 25-38.

Pimentel G., Rees C., Souza P. DE \& Ayala P. 2010. — Estrategias de movilidad del período formativo en la depresión intermedia, desierto de Atacama, in Actas del XVII Congreso Nacional de Arqueologia Chilena, Tomo II. Kultrún, Valdivia: 1353-1364.

Pimentel G., Rees C., SouZa P. De \& Arancibia L. 2011. Viajeros costeros y caravaneros. Dos estrategias de movilidad en el Período Formativo del Desierto de Atacama, Chile, in Núñez L. \& Nielsen A. (eds), En Ruta. Arqueología, Historia y Etnografía del Tráfico Sur Andino. Encuentro Grupo Editor, Córdoba: 43-81.

Pimentel G., Ugarte M., Blanco J., Torres-Rouff C. \& Pestle W. 2017. - Calate. De lugar desnudo a laboratorio arqueológico de la movilidad y el tráfico intercultural prehispánico en el desierto de Atacama (ca. 7000 AP-550 AP). Estudios Atacameños 56: 23-58.

Pollard G. 1971. - Cultural change and adaptation in the Central Atacama Desert of northern Chile. Nawpa Pacha: Journal of Andean Archaeology 9: 41-64.

Rees C. \& Souza P. DE 2004. - Producción lítica durante el Período Formativo en la cuenca alta del río Salado. Chungara, Revista de Antropología Chilena Volumen Especial: 619-639.

REINHARD K. 1992. - Parasitology as an interpretive tool in archaeology. American Antiquity 57 (2): 231-245.

Reinhard K. \& Urban O. 2003. - Diagnosing ancient Diphyllobothriasis from Chinchorro mummies. Memórias do Instituto Oswaldo Cruz 98 (1): 191-193. https://doi.org/10.1590/S007402762003000900028

Rivera M. 2002. - Historias del Desierto: arqueología del Norte de Chile. Editorial del Norte, La Serena, 238 p.

Rivera M., Shea D., Carevic A. \& GrafFam G. 1996. — En torno a los orígenes de las sociedades complejas andinas: excavaciones en Ramaditas, una aldea Formativa del Desierto de Atacama, Chile. Diálogo Andino 14-15: 205-239.

ROSARIO B. 1970. - Nota sobre la alimentación del "jurel” (Trachurus murphy Nichols). Noticiario mensual Museo nacional de Historia natural (167): 6-8.

Salazar D., Castro V., Michelow J., Salinas H., Figueroa V. \& Mille B. 2010. - Minería y metalurgia en la costa arreica de la región de Antofagasta, norte de Chile. Boletín del Museo Chileno de Arte Precolombino 15 (1): 9-23.

SANTANa F. 2011. - Multiculturalidad en el Cementerio Oriente de Quillagua;Co-existencia de grupos culturales? Una aproximación desde la bioantropología mediante análisis isotópicos de dieta y movilidad en el curso inferior del río Loa, Periodo Intermedio Tardío. Degree dissertation, Universidad de Chile, Santiago, 98 p.

Santana F., Herrera M. \& Uribe M. 2012. - Acercamiento a la paleodieta en la costa y quebradas tarapaqueńas durante el periodo Formativo: análisis de isótopos estables a partir de tres casos de estudio. Boletín de la Sociedad Chilena de Arqueología 41-42: 109-126.

Santana F., Hubbe M. \& Uribe M. 2015. — Isotopic evidence for marine consumption and mobility in the Atacama Desert (Quillagua, northern Chile). International Journal of Osteoarchaeology. https://doi.org/10.1002/oa.2437

SANTORO C., VinTON S. \& ReINHARD K. 2003. - Inca expansion and parasitism in the Lluta Valley: preliminary data. Memórias do Instituto Oswaldo Cruz 98 (1): 161-163.

SCOTt L., NePstad-Thornberry C. \& Puseman K. 2005. - Restos paleofecales del sitio de Ramaditas, Norte de Chile: aspectos sobre dieta y salud en el Formativo Medio y Tardío, in RIVERA M. (ed.), Arqueología del Desierto de Atacama. La etapa Formativa en el área de Ramaditas/Guatacondo. Universidad Bolivariana, Santiago: 195-210.

Silva A. \& Oliva M. 2010. - Revisión sobre aspectos biológicos y de cultivo del lenguado chileno (Paralichthys adspersus). Latin American Journal of Aquatic Research 38 (3): 377-386. 
Silva J. \& BAHAMONDES R. 1968. - La potera, anzuelo para cefalópodos. Anales del Museo de Historia Natural de Valparaiso 1: 217-237.

SinClaire C. 2008. - Un antiguo chango de Mejillones, in BereNGUER J. (ed.), Pescadores en la Niebla, los changos y sus ancestros. Museo Chileno de Arte Precolombino, Santiago: 80-83.

SMith M. 2006. - The Archaeology of Food Preference. American Anthropologist 108 (3): 480-493.

SOUZA P. DE 2004. - Tecnologías de proyectil durante los períodos Arcaico y Formativo en el Loa superior (Norte de Chile): a partir del análisis de puntas líticas. Chungara, Revista de Antropología Chilena 36 Suppl. 1: 61-76. https://doi.org/10.4067/S071773562004000300009

Souza P. De, Cartajena I., NúÑEz L. \& Carrasco C. 2010. Cazadores-recolectores del arcaico tardío y desarrollo de complejidad social en la puna de Atacama: las evidencias del sitio Tulán-52 (norte árido de Chile). Werkén 13 (2): 91-118.

SPAHNI J. 1967. - Recherches archéologiques à l'embouchure du Rio Loa (Côte du Pacifique - Chili). Journal de la Société des Américanistes 56 (1): 181-239.

Stuiver M., Reimer P. \& Reimer R. 2005. - CALIB 14C Calibration Program version 5.0. http://intcal.qub.ac.uk/calib/manual/ index, last consultation: 04/03/2019.

Thomas C., Benavente A., Cartajena I. \& Serracino G. 1994. Topater, un cementerio temprano: una aproximación simbólica, in Actas del XIII Congreso Nacional de Arqueología Chilena, Tomo II. Universidad de Antofagasta, Antofagasta: 159-173.

Torres-Rouff C., Pestle W. \& Gallardo F. 2012a. — Eating fish in the driest desert in the world: osteological and biogeochemical analyses of human skeletal remains from the San Salvador cemetery, north Chile. Latin American Antiquity 23 (1): 51-69. https://doi.org/10.7183/1045-6635.23.1.51

Torres-Rouff C., Pimentel G. \& UGarte M. 2012b. —¿QQuiénes viajaban? Investigando la muerte de viajeros prehispánicos en el desierto de Atacama (ca. 800 AC-1536 DC). Estudios Atacameños 43: 167-186.

Urbina S., AdÁn L. \& Pellegrino C. 2012. - Arquitecturas formativas de las quebradas de Guatacondo y Tarapacá a través del proceso aldeano (ca. 900 AC-1000 DC). Boletín del Museo Chileno de Arte Precolombino 17 (1):31-60. https://doi.org/10.4067/ S0718-68942012000100003

URIBE M. 2009. - El período Formativo de Tarapacá y su cerámica: Avances sobre complejidad social en la costa del Norte Grande de Chile (900 A.C.-800 D.C.). Estudios Atacameños 37: 5-27. https://doi.org/10.4067/S0718-10432009000100002

Uribe M. \& AyAla P. 2004. — La alfarería de Quillagua en el contexto formativo del Norte Grande de Chile (1000 A.C.500 D.C.). Chungara, Revista de Antropología Chilena Volumen Especial: 585-598.

Uribe M. \& Vidal E. 2012. - Sobre la secuencia cerámica del Período Formativo de Tarapacá (900 A.C.-900 D.C.): Estudios en Pircas, Caserones, Guatacondo y Ramaditas, Norte de Chile. Chungara, Revista de Antropología Chilena 44 (2): 209-245.

VAÏsse E., Hoyos F. \& ECHEVERría I ReYes A. 1896. - Glosario de la lengua Atacameña. Imprenta Cervantes, Santiago, 36 p.

VAn DeR Veen M. 2003. - When is food a luxury? World Archaeology 34 (3): 405-427.

Van Kessel J. 1988. - Diccionario de pesca artesanal del Norte Grande de Chile. Universidad Libre de Amsterdam; Centro de Investigaciones de la Realidad del Norte, Iquique, $254 \mathrm{p}$.

Vargas L., Falabella F. \& Meléndez R. 1993. - Bases para el manejo de datos ictioarqueológicos del "jurel" (Trachurus symmetricus Ayres, 1855) (Pisces: Perciformes: Carangidae). Boletín del Museo Regional de la Araucania 4: 355-371.

WEISCHET W. 1975. — Las condiciones climáticas del desierto de Atacama como desierto extremo de la tierra. Norte Grande 1 (3-4): 363-373.

WiLliams L. 1980. - Analysis of coprolites from six sites in northern Chile, in Meighan C. \& True D. (eds), Prehistoric Trails of Atacama: Archaeology of Northern Chile. The Institute of Archaeology of the University of California, Los Angeles: 195-204.

WoOdBURN J. 1982. — Egalitarian societies. Man 17 (3): 431-451.

YAÑEZ P. 1955. - Peces útiles de la costa chilena. Revista de Bilogía Marina 6: 29-81.

ZLATAR V. 1975. - Estructura habitacionales del sitio Caleta Huelén 42. [unpublished]. 